\title{
Time-resolved spectral analysis of the pulsating helium star V652 Her $^{\star}$
}

\author{
C. S. Jeffery ${ }^{1}$, V. M. Woolf ${ }^{1}$, and D. L. Pollacco ${ }^{2}$ \\ 1 Armagh Observatory, College Hill, Armagh BT61 9DG, Northern Ireland \\ 2 School of Physical Sciences, Queen's University Belfast, Belfast BT7 9NN, Northern Ireland
}

Received 19 January 2001 / Accepted 27 June 2001

\begin{abstract}
A series of 59 moderate-resolution high signal-to-noise spectra of the pulsating helium star V652 Her covering 1.06 pulsation cycles was obtained with the William Herschel Telescope. These have been supplemented by archival ultraviolet and visual spectrophotometry and used to make a time-dependent study of the properties of V652 Her throughout the pulsation cycle. This study includes the following features: the most precise radial velocity curve for V652 Her measured so far, new software for the automatic measurement of effective temperature, surface gravity and projected rotation velocities from moderate-resolution spectra, self-consistent high-precision measurements of effective temperature and surface gravity around the pulsation cycle, a demonstration of excessive line-broadening at minimum radius and evidence for a pulsation-driven shock front, a new method for the direct measurement of the radius of a pulsating star using radial velocity and surface gravity measurements alone, new software for the automatic measurement of chemical abundances and microturbulent velocity, updated chemical abundances for V652 Her compared with previous work (Paper IV), a reanalysis of the total flux variations (cf. Paper II) in good agreement with previous work, and revised measurements of the stellar mass and radius which are similar to recent results for another pulsating helium star, BX Cir. Masses measured without reference to the ultraviolet fluxes turn out to be unphysically low $\left(\sim 0.18 \quad M_{\odot}\right)$. The best estimate for the dimensions of V652 Her averaged over the pulsation cycle is given by: $\left\langle T_{\text {eff }}\right\rangle=22930 \pm 10 \mathrm{~K}$ and $\langle\log g\rangle=3.46 \pm 0.05$ (ionization equilibrium), $\left\langle T_{\text {eff }}\right\rangle=20950 \pm 70 \mathrm{~K}$ (total flux method), $\langle R\rangle=2.31 \pm 0.02 R_{\odot},\langle L\rangle=919 \pm 14 L_{\odot}$, $M=0.59 \pm 0.18 M_{\odot}$ and $d=1.70 \pm 0.02 \mathrm{kpc}$. Two significant problems were encountered. The line-blanketed hydrogen-deficient model atmospheres used yield effective temperatures from the optical spectrum (ionization equilibrium) and visual and UV photometry (bolometric flux) that are inconsistent. Secondly, the IUE spectra are poorly distributed in phase and have low signal-to-noise. These problems may introduce systematic errors of up to $0.1 M_{\odot}$.
\end{abstract}

Key words. stars: fundamental parameters - stars: abundances - stars: individual: V652 Her - stars: oscillations - techniques: spectroscopic

\section{Introduction}

The early-type helium star V652 Her pulsates with a period of 0.108 days (Landolt 1975), a visual amplitude of $0.1 \mathrm{mag}$ and a radial velocity amplitude of $\sim 70 \mathrm{~km} \mathrm{~s}^{-1}$ (Hill et al. 1981, Paper I). These properties have made it amenable to detailed analyses, notably the direct measurement of its average radius $\left(R=1.98 R_{\odot}\right.$, Lynas Gray et al. 1984, Paper II), leading to precise estimates of its mass $\left(M=0.7 \quad M_{\odot}\right)$ and luminosity (Paper II; Jeffery et al. 1999, Paper IV). Added to these data is the observation that the pulsation period is decreasing at a rate

Send offprint requests to: C. S. Jeffery,

e-mail: csj@star.arm.ac.uk

* Based on observations obtained with the William Herschel Telescope, the United Kingdom Infrared Telescope, and on INES data from the IUE satellite. commensurate with a secular contraction (Kilkenny \& Lynas-Gray 1982; Kilkenny \& Lynas-Gray 1984; Kilkenny 1988; Kilkenny et al. 1996). Such statistics have enabled the evolutionary status and pulsational properties of the star to be investigated using theoretical models. Both pose serious challenges to stellar structure theory.

In the case of pulsations, the instability of V652 Her could only be understood correctly when the contribution of iron-group opacities at around $10^{5} \mathrm{~K}$ was correctly taken into account (Saio 1993). Models with $R=2 R_{\odot}$ and $M=0.7 M_{\odot}$ now reproduce the pulsational properties of V652 Her (Fadeyev \& Lynas-Gray 1996) very well.

The evolutionary status of V652 Her also poses a conundrum. Neither of the principal candidates for the progeny of carbon-rich extreme helium stars is satisfactory because the surface of V652 Her consists entirely of a CNO-processed material. A substantial yield of surface 
carbon is anticipated from both the merger of a $\mathrm{CO}$ with a He white dwarf (Webbink 1984; Iben \& Tutukov 1985) and from a late helium shell-flash in a $\mathrm{CO}$ white dwarf (Iben et al. 1983; Iben \& McDonald 1995). Models derived from both scenarios also predict evolutionary tracks that are overluminous compared with V652 Her. However, in contrast to the merger of a $\mathrm{CO}$ and He white dwarf, recent models for the merger of two helium white dwarfs (Saio \& Jeffery 2000) have successfully reproduced nearly all of the observed properties of V652 Her.

Consequently, V652 Her provides a very important test for stellar evolution and pulsation theory. Being comparatively bright, it may also allow sensitive tests of the physics of stellar pulsation and evolution and of radiative transfer in pulsating star atmospheres. The observations presented in this paper were obtained in order to significantly refine results obtained previously. They have also precipitated the development of powerful new tools for the analysis of stellar spectra.

\section{Observations}

\subsection{High resolution optical spectroscopy}

Observations were made with the William Herschel Telescope of the Isaac Newton Group on La Palma during 3 hours of service time on the night of 1998 July 16. Spectra were obtained using a 1200 line/mm grating and the EEV10 CCD on the blue arm of ISIS and a slit-width of $0.7^{\prime \prime}$ yielding a nominal (2 pixel) resolution $R=10000$. Repeated integrations of $100 \mathrm{~s}$ yielded 59 spectra of the target, with a typical signal-to-noise $(1 \sigma)$ ratio of 100 in each spectrum. Integrations were interrupted every $40 \mathrm{~min}$ to obtain comparison spectra of a copper-argon lamp. The journal of observations is reflected by the heliocentric Julian times given in Table 1 . These times have been converted to pulsation phase using the most recent quartic ephemeris (Kilkenny et al. 1996).

The stellar spectra were bias subtracted, flatfielded, sky subtracted and optimally extracted to one dimension using FIGARO data reduction software. The comparison lamp frames were interpolated linearly in time to provide a wavelength calibration frame corresponding to each stellar observation. Both stellar spectra and the interpolated comparison lamp spectra were calibrated and linearized in order to provide an independent check of the subsequent velocity measurements.

Each individual stellar spectrum was normalized with respect to the local continuum. This local continuum was defined, for each spectrum, by fitting an 11th order polynomial to data defined by 23 spectral windows judged to be, as far as possible, free of significant absorption lines. A further correction to the continuum was applied during the spectral fitting procedure discussed in Appendix A.

\subsection{IUE and visual spectrophotometry}

Although the high-resolution spectroscopy is sufficient to make a completely self-consistent and independent measurement of the mass of V652 Her, important additional information is available from optical and ultraviolet spectrophotometry.

Johnson $V$-band photometry (Kilkenny \& Lynas-Gray 1982) were used in two ways. In the first instance they were combined with effective temperatures measured spectroscopically to provide measurements of angular diameter at much higher phase resolution. In the second they were combined with Strömgren photometry (also from Kilkenny \& Lynas-Gray 1982) and with ultraviolet data to provide phase-resolved spectrophotometry effectively covering the wavelength interval 1150 to $5500 \AA$.

Several spectra of V652 Her were obtained with IUE (cf. Paper II). All available spectra were downloaded from the IUE Newly-Extracted Spectra (INES, Rodriguez-Pascual et al. 1999) archive web server in February 2000. The IUE data do not sample V652 Her's pulsation cycle evenly. We analyzed the data where we could find SWP (1150 $<<<1980 \AA)$ and LWR $(1850 \AA<\lambda<3350 \AA)$ spectra measured at similar phases. The phase bins chosen and the IUE spectra used are listed in Table 3 in Sect. 4. The phases listed for each spectrum were calculated using the ephemeris of Kilkenny et al. (1996). When preparing the spectra we used the SWP data in the region where SWP and LWR spectra overlap in wavelength.

\subsection{Infrared spectroscopy}

A low dispersion $J$-band spectrum of V652 Her was obtained using a cooled grating spectrometer (CGS4) on the United Kingdom Infrared Telescope (UKIRT) on 2000 June 7 . With a 2-pixel spectral resolution of $\approx 900$, the entire interval from 0.98 to $1.23 \mu \mathrm{m}$. As far as we know, this is the first infrared spectrum of a B-type extreme helium star to be published. The data are presented and discussed briefly in Sect. 5 .

\section{Radial velocities}

Radial velocities $\left(v_{i}\right)$ were measured by cross-correlating individual spectra with respect to a template spectrum. The procedure included the subtraction of the continuum and the conversion of the wavelength scale to logarithmic units, before calculation of the crosscorrelation function (ccf). Several strong lines, namely He I $\lambda 4388,4471,4921 \AA, \mathrm{H} \beta$ and $\mathrm{H} \gamma$, were excluded. The ccf was then converted to velocity units and the position and width of its peak were measured by fitting a Gaussian to those data within the peak having values between 0.3 and 1.0 .

The exercise was repeated using the interpolated comparison lamp spectra. Small systematic shifts were observed within each continuous run, with larger shifts 
Table 1. Observational data for V652 Her, including WHT Run number, heliocentric Julian time of observation (HJD), pulsation phase $(\phi)$ according to the quartic ephemeris of Kilkenny et al. (1996), heliocentric radial velocity $(v)$, astrocentric radial velocity $(\dot{r})$ assuming $p$ from Eq. (4), radial displacement $(\delta r)$, surface accleration $(\ddot{r})$, effective temperature $\left(T_{\text {eff }}\right)$, effective surface gravity $\left(\log g_{\text {eff }}\right)$, and true gravity at stellar surface $(\log g)$. Around $\phi \sim 0.15$, the sum $g_{\text {eff }}-\ddot{r}$ becomes negative for reasons discussed in Sect. 6, hence $\log g=-\mathrm{NaN}$.

\begin{tabular}{|c|c|c|c|c|c|c|c|c|c|c|c|c|}
\hline Run & $\begin{array}{l}\text { HJD - } \\
2451010\end{array}$ & $\phi$ & $\begin{array}{r}v \\
\mathrm{kms}^{-1}\end{array}$ & \pm & $\begin{array}{r}\dot{r} \\
\mathrm{kms} \mathrm{s}^{-1}\end{array}$ & $\begin{array}{c}\delta r \\
10^{5} \mathrm{~km}\end{array}$ & $\mathrm{~km} \mathrm{~s}^{-2}$ & $\mathrm{kK}^{T_{\mathrm{c}}}$ & \pm & $\begin{array}{r}\log \\
(\mathrm{cgs})\end{array}$ & \pm & $\begin{array}{l}\log g \\
(\mathrm{cgs})\end{array}$ \\
\hline 486 & 0.4376 & 0.107 & 34.94 & 1.11 & -38.74 & -1.191 & 0.125 & 25.23 & 0.09 & 3.61 & 0.01 & $-\mathrm{NaN}$ \\
\hline 487 & 0.4394 & 0.123 & 21.17 & 0.76 & -19.61 & -1.235 & 0.163 & 25.10 & 0.10 & 3.72 & 0.01 & $-\mathrm{NaN}$ \\
\hline 488 & 0.4412 & 0.140 & -0.86 & 0.81 & 11.20 & -1.242 & 0.171 & 25.19 & 0.10 & 3.76 & 0.01 & $-\mathrm{NaN}$ \\
\hline 489 & 0.4430 & 0.157 & -16.55 & 1.29 & 33.03 & -1.208 & 0.101 & 25.18 & 0.10 & 3.75 & 0.01 & $-\mathrm{NaN}$ \\
\hline 490 & 0.4448 & 0.173 & -23.27 & 1.07 & 42.34 & -1.149 & 0.040 & 24.88 & 0.09 & 3.59 & 0.01 & $-\mathrm{NaN}$ \\
\hline 491 & 0.4466 & 0.190 & -25.52 & 0.94 & 45.45 & -1.082 & 0.014 & 25.17 & 0.09 & 3.57 & 0.01 & 3.36 \\
\hline 492 & 0.4483 & 0.207 & -26.38 & 0.97 & 46.64 & -1.011 & 0.002 & 24.74 & 0.09 & 3.46 & 0.01 & 3.44 \\
\hline 493 & 0.4501 & 0.223 & -25.86 & 0.74 & 45.92 & -0.940 & -0.005 & 24.69 & 0.09 & 3.42 & 0.01 & 3.49 \\
\hline 494 & 0.4519 & 0.240 & -25.30 & 0.78 & 45.15 & -0.870 & -0.004 & 24.10 & 0.09 & 3.33 & 0.01 & 3.42 \\
\hline 495 & 0.4537 & 0.256 & -24.87 & 0.68 & 44.56 & -0.801 & -0.009 & 23.81 & 0.09 & 3.38 & 0.01 & 3.52 \\
\hline 497 & 0.4569 & 0.286 & -22.43 & 0.83 & 41.18 & -0.682 & -0.011 & 23.68 & 0.09 & 3.39 & 0.01 & 3.56 \\
\hline 498 & 0.4587 & 0.303 & -21.29 & 0.39 & 39.61 & -0.620 & -0.012 & 22.92 & 0.09 & 3.31 & 0.01 & 3.51 \\
\hline 499 & 0.4604 & 0.319 & -19.87 & 0.64 & 37.63 & -0.561 & -0.014 & 22.81 & 0.09 & 3.36 & 0.01 & 3.57 \\
\hline 500 & 0.4622 & 0.336 & -18.10 & 0.61 & 35.18 & -0.505 & -0.017 & 23.03 & 0.09 & 3.27 & 0.01 & 3.55 \\
\hline 501 & 0.4640 & 0.352 & -16.14 & 0.58 & 32.46 & -0.453 & -0.014 & 22.53 & 0.09 & 3.25 & 0.01 & 3.50 \\
\hline 502 & 0.4658 & 0.369 & -15.02 & 0.60 & 30.90 & -0.404 & -0.010 & 22.44 & 0.09 & 3.31 & 0.01 & 3.49 \\
\hline 503 & 0.4675 & 0.386 & -13.87 & 0.64 & 29.32 & -0.358 & -0.009 & 22.20 & 0.08 & 3.37 & 0.01 & 3.50 \\
\hline 504 & 0.4693 & 0.402 & -13.10 & 0.62 & 28.25 & -0.314 & -0.010 & 22.12 & 0.08 & 3.23 & 0.01 & 3.43 \\
\hline 505 & 0.4711 & 0.419 & -11.69 & 0.70 & 26.28 & -0.272 & -0.009 & 22.31 & 0.09 & 3.26 & 0.01 & 3.44 \\
\hline 506 & 0.4729 & 0.435 & -11.12 & 0.75 & 25.50 & -0.232 & -0.012 & 21.96 & 0.08 & 3.26 & 0.01 & 3.48 \\
\hline 507 & 0.4748 & 0.454 & -8.83 & 0.42 & 22.30 & -0.192 & -0.015 & 21.75 & 0.08 & 3.21 & 0.01 & 3.49 \\
\hline 509 & 0.4780 & 0.483 & -6.37 & 0.45 & 18.87 & -0.135 & -0.014 & 21.81 & 0.08 & 3.26 & 0.01 & 3.51 \\
\hline 510 & 0.4798 & 0.500 & -4.58 & 0.74 & 16.39 & -0.108 & -0.015 & 21.51 & 0.08 & 3.20 & 0.01 & 3.49 \\
\hline 511 & 0.4816 & 0.516 & -3.01 & 0.74 & 14.20 & -0.085 & -0.013 & 21.64 & 0.08 & 3.22 & 0.01 & 3.48 \\
\hline 512 & 0.4833 & 0.533 & -1.63 & 0.69 & 12.27 & -0.065 & -0.011 & 21.76 & 0.08 & 3.23 & 0.01 & 3.46 \\
\hline 513 & 0.4851 & 0.549 & -0.50 & 0.68 & 10.68 & -0.047 & -0.008 & 21.58 & 0.08 & 3.23 & 0.01 & 3.40 \\
\hline 514 & 0.4869 & 0.566 & 0.13 & 0.68 & 9.81 & -0.031 & -0.010 & 21.29 & 0.08 & 3.20 & 0.01 & 3.42 \\
\hline 515 & 0.4887 & 0.582 & 1.76 & 0.71 & 7.52 & -0.018 & -0.014 & 21.38 & 0.08 & 3.20 & 0.01 & 3.48 \\
\hline 516 & 0.4904 & 0.599 & 3.31 & 0.52 & 5.36 & -0.008 & -0.017 & 21.28 & 0.08 & 3.22 & 0.01 & 3.53 \\
\hline 517 & 0.4922 & 0.616 & 5.57 & 0.46 & 2.19 & -0.002 & -0.015 & 21.41 & 0.08 & 3.15 & 0.01 & 3.47 \\
\hline 518 & 0.4940 & 0.632 & 6.65 & 0.68 & 0.68 & 0.000 & -0.016 & 21.27 & 0.08 & 3.22 & 0.01 & 3.52 \\
\hline 519 & 0.4958 & 0.649 & 9.18 & 0.48 & -2.87 & -0.002 & -0.010 & 21.32 & 0.08 & 3.21 & 0.01 & 3.42 \\
\hline 523 & 0.5016 & 0.703 & 11.49 & 0.70 & -6.09 & -0.024 & -0.008 & 21.36 & 0.08 & 3.22 & 0.01 & 3.39 \\
\hline 524 & 0.5034 & 0.720 & 12.83 & 0.69 & -7.97 & -0.035 & -0.007 & 21.62 & 0.08 & 3.26 & 0.01 & 3.39 \\
\hline 525 & 0.5052 & 0.736 & 12.99 & 0.67 & -8.20 & -0.048 & -0.015 & 21.31 & 0.08 & 3.24 & 0.01 & 3.51 \\
\hline 526 & 0.5070 & 0.753 & 16.10 & 0.68 & -12.55 & -0.064 & -0.021 & 21.26 & 0.08 & 3.18 & 0.01 & 3.56 \\
\hline 527 & 0.5087 & 0.770 & 17.58 & 0.73 & -14.61 & -0.084 & -0.014 & 21.30 & 0.08 & 3.19 & 0.01 & 3.46 \\
\hline 528 & 0.5105 & 0.786 & 19.07 & 0.48 & -16.70 & -0.108 & -0.020 & 21.43 & 0.08 & 3.17 & 0.01 & 3.54 \\
\hline 529 & 0.5123 & 0.803 & 21.89 & 0.44 & -20.61 & -0.137 & -0.023 & 21.86 & 0.08 & 3.16 & 0.01 & 3.58 \\
\hline 530 & 0.5141 & 0.819 & 24.24 & 0.74 & -23.89 & -0.171 & -0.019 & 21.61 & 0.08 & 3.21 & 0.01 & 3.55 \\
\hline 531 & 0.5159 & 0.836 & 26.05 & 0.72 & -26.41 & -0.210 & -0.012 & 21.81 & 0.08 & 3.23 & 0.01 & 3.46 \\
\hline 532 & 0.5176 & 0.852 & 26.92 & 0.67 & -27.62 & -0.251 & -0.004 & 21.90 & 0.08 & 3.19 & 0.01 & 3.29 \\
\hline 533 & 0.5194 & 0.869 & 26.94 & 0.64 & -27.64 & -0.294 & -0.010 & 22.16 & 0.08 & 3.28 & 0.01 & 3.47 \\
\hline
\end{tabular}


Table 1. continued.

\begin{tabular}{|l|l|l|rr|r|r|r|rr|rr|r|}
\hline Run & $\begin{array}{l}\text { HJD }- \\
\end{array}$ & $\phi$ & $\begin{array}{r}v \\
241010\end{array}$ & & $\mathrm{~km} \mathrm{~s}^{-1}$ & \pm & $\begin{array}{r}\dot{r} \\
\mathrm{~km} \mathrm{~s}^{-1}\end{array}$ & $\begin{array}{r}\delta r \\
10^{5} \mathrm{~km}\end{array}$ & $\begin{array}{r}\dot{r} \\
\mathrm{~km} \mathrm{~s}^{-2}\end{array}$ & $\begin{array}{r}T_{\text {eff }} \\
\mathrm{kK}\end{array}$ & $\begin{array}{c}\log g_{\mathrm{eff}} \\
(\mathrm{cgs})\end{array}$ & $\begin{array}{c}\log g \\
(\mathrm{cgs})\end{array}$ \\
\hline 535 & 0.5235 & 0.907 & 30.69 & 0.67 & -32.85 & -0.400 & -0.013 & 22.92 & 0.09 & 3.29 & 0.01 & 3.51 \\
536 & 0.5253 & 0.923 & 31.59 & 0.65 & -34.10 & -0.451 & -0.010 & 22.74 & 0.09 & 3.35 & 0.01 & 3.52 \\
537 & 0.5270 & 0.940 & 32.97 & 0.67 & -36.01 & -0.505 & -0.018 & 23.80 & 0.09 & 3.36 & 0.01 & 3.61 \\
538 & 0.5288 & 0.957 & 35.49 & 0.85 & -39.50 & -0.564 & -0.014 & 24.00 & 0.09 & 3.36 & 0.01 & 3.57 \\
539 & 0.5306 & 0.973 & 36.04 & 0.86 & -40.26 & -0.625 & -0.006 & 24.53 & 0.09 & 3.41 & 0.01 & 3.50 \\
540 & 0.5324 & 0.990 & 36.80 & 0.81 & -41.32 & -0.687 & -0.012 & 24.60 & 0.09 & 3.39 & 0.01 & 3.56 \\
541 & 0.5341 & 1.006 & 38.65 & 0.72 & -43.88 & -0.753 & -0.018 & 24.75 & 0.09 & 3.38 & 0.01 & 3.62 \\
542 & 0.5359 & 1.023 & 40.73 & 0.62 & -46.75 & -0.822 & -0.008 & 25.49 & 0.08 & 3.49 & 0.01 & 3.60 \\
543 & 0.5377 & 1.039 & 40.52 & 0.65 & -46.46 & -0.894 & -0.001 & 25.60 & 0.08 & 3.56 & 0.01 & 3.57 \\
544 & 0.5395 & 1.056 & 40.92 & 0.56 & -47.01 & -0.966 & -0.015 & 25.74 & 0.08 & 3.50 & 0.01 & 3.67 \\
545 & 0.5413 & 1.072 & 43.79 & 0.50 & -50.97 & -1.041 & 0.013 & 25.40 & 0.09 & 3.57 & 0.01 & 3.37 \\
547 & 0.5444 & 1.102 & 36.77 & 0.64 & -41.27 & -1.167 & 0.054 & 25.99 & 0.09 & 3.62 & 0.01 & $-\mathrm{NaN}$ \\
548 & 0.5462 & 1.118 & 27.11 & 1.04 & -27.88 & -1.220 & 0.140 & 25.24 & 0.10 & 3.72 & 0.01 & $-\mathrm{NaN}$ \\
549 & 0.5480 & 1.135 & 5.90 & 0.35 & 1.73 & -1.240 & 0.183 & 25.47 & 0.10 & 3.83 & 0.01 & $-\mathrm{NaN}$ \\
550 & 0.5497 & 1.151 & -13.21 & 1.32 & 28.40 & -1.217 & 0.123 & 25.74 & 0.10 & 3.90 & 0.01 & $-\mathrm{NaN}$ \\
551 & 0.5515 & 1.168 & -21.16 & 0.97 & 39.43 & -1.165 & 0.072 & 25.10 & 0.09 & 3.64 & 0.01 & $-\mathrm{NaN}$ \\
\hline
\end{tabular}

occurring between runs (Fig. 1). In principle, these shifts could have been applied to the velocity data. However they are substantially smaller than the formal errors in the stellar velocities, and their origin is not entirely clear.

The template adopted for the stellar ccf's was the spectrum for run 518, corresponding to $\phi=0.633$ and very close to maximum radius (Paper II). Here, the photosphere is stationary and line distortions introduced by the center-to-limb difference in the projected radial velocity are minimal. The radial velocity of the template $v_{\mathrm{T}}$ was measured initially by cross-correlation with a synthetic spectrum computed for the (stationary) model atmosphere described in Paper IV, and subsequently with the best-fit synthetic spectrum described in Sect. 4. The individual and template velocities were combined and corrected to the heliocentric frame, $v_{i \odot}=v_{i}+v_{\mathrm{T}}-v_{\oplus}$, where $v_{\oplus}$ is the correction for the earth motion. The results are shown in Table 1 and in Fig. 1. The errors shown are the formal errors in $v_{\mathrm{i}}$ given by the least squares Gaussian fit to the ccf peaks. The error in $v_{\mathrm{T}}$ is $\pm 0.92 \mathrm{~km} \mathrm{~s}^{-1}$.

The mean or systemic radial velocity $\left(v_{\star}\right)$ is determined by the condition that the integral of the velocity relative to the center of mass over a complete pulsation cycle must vanish:

$\int_{0}^{1}\left(v_{0}-v_{\star}\right) \frac{\mathrm{d} t}{\mathrm{~d} \phi} \mathrm{d} \phi=0$.

From the current data we find $v_{\star}=7.14 \pm 0.92 \mathrm{~km} \mathrm{~s}^{-1}$. This result is independent of the velocity projection factor (Sect. 6) and the error is dominated by the measurement of $v_{\mathrm{T}}$. It is tempting to compare this result with that of (Jeffery \& Hill 1986, Paper III), where systemic velocities of $1.25 \mathrm{~km} \mathrm{~s}^{-1}$ and $3.51 \mathrm{~km} \mathrm{~s}^{-1}$ were deduced from data obtained from 1979-1982 and in 1984 respectively. However it may be premature to suggest that these differences represent any more than major improvements in detector and measurement technology; the earliest data were calibrated against an F-star velocity standard and the 1984 data were calibrated by fitting parabolae to selected lines in a template spectrum which had been integrated over several pulsation cycles.

A cursory examination of the spectra as a function of phase quickly demonstrated large changes in both the strengths and widths of several lines, particularly around phases corresponding to minimum radius. The variations in line width are reflected in the widths of the ccfs measured above (Fig. 1). Several line-broadening mechanisms are in operation and are discussed below (Sect. A.5).

\section{Temperatures, gravities and abundances}

The analysis of such a series of spectra entails fitting a large quantity of data with theoretical models that have many free parameters including effective temperature $\left(T_{\text {eff }}\right)$, surface gravity $(\log g)$, microturbulent and rotational velocity $\left(v_{\mathrm{t}}, v \sin i\right)$ and elemental abundances $(n)$. This fitting has been achieved through the development of a suite of modelling and fitting programs, including STERNE, SPECTRUM, LTE_LINES, FFIT, SFIT and SFIT_SYNTH. These are described more fully in Appendix A.

\subsection{The model grid}

Using STERNE and SPECTRUM, grids of model atmospheres, spectral energy distributions and high-resolution synthetic spectra were calculated for use with the fitting programs. After iteration, the final composition adopted as input for the grid of model atmospheres and synthetic spectra comprised $n_{\mathrm{H}}=0.01, n_{\mathrm{He}}=0.99, n_{\mathrm{C}}=$ 0.00004 and $n_{\mathrm{N}}=0.0025$. All other elements were assumed to have solar-like relative abundances. The grid covered the ranges $T_{\text {eff }}=17000(1000) 30000 \mathrm{~K}$ and $\log g=2.75(0.25) 4.25$ (cgs). 

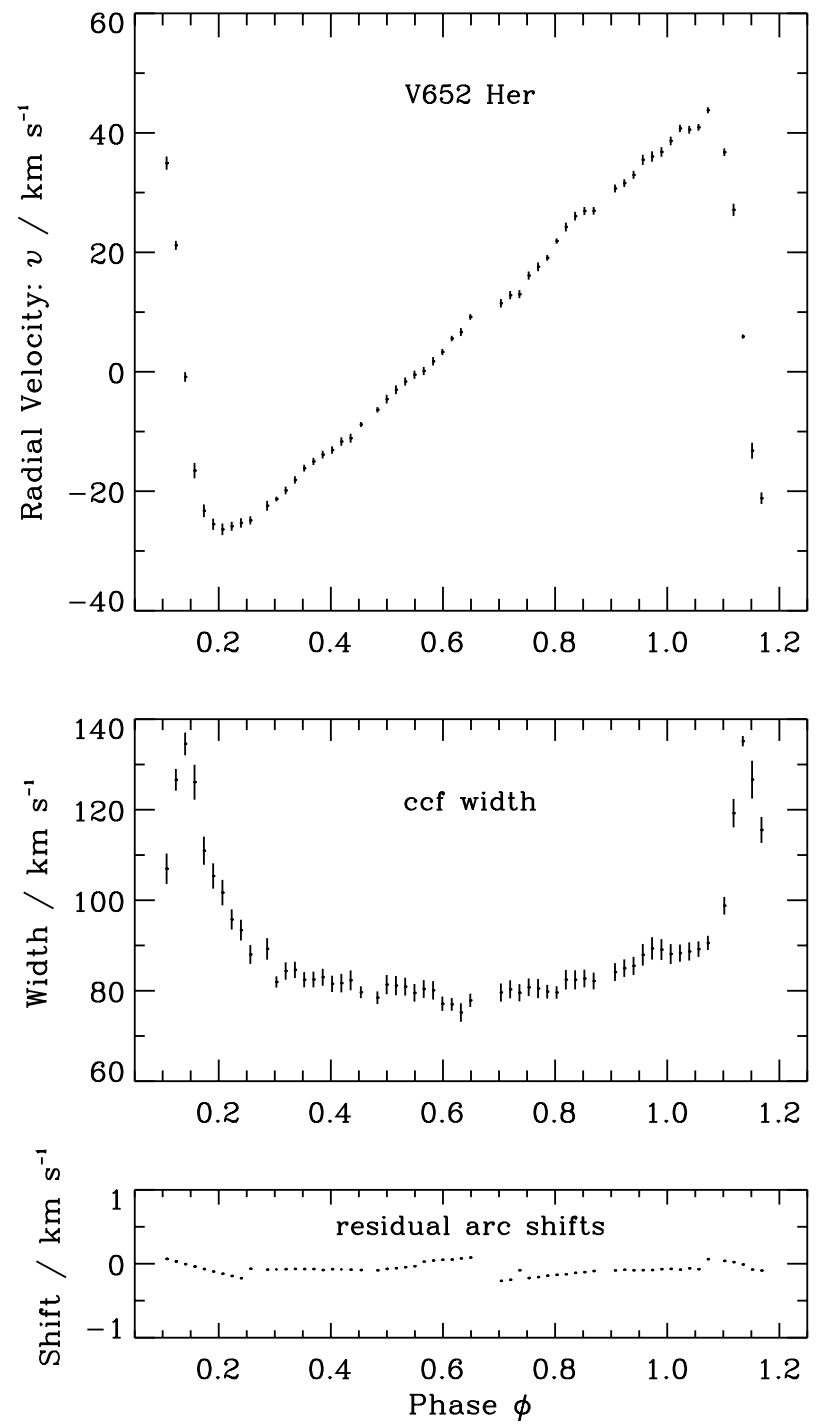

Fig. 1. The radial velocity curve of V652 Her (top). Vertical lines represent the errors from Table 1. Also shown are the absorption line widths as represented by the ccf width (FWHM, middle) and the residual shifts of the interpolated comparison arcs (bottom).

\section{2. $T_{\text {eff }}, \log g_{\text {eff }}, v \sin i$}

Procedure SFIT was applied to the 1998 spectroscopy. The central $2 \AA$ of each of the strongest He I lines was excluded from the $\chi^{2}$ minimization because current helium-rich model atmospheres consistently fail to match the cores of these lines. This is true for both variable and non-variable stars (cf. Heber 1983). Note that for a pulsating star, this procedure measures the effective surface gravity $\left(g_{\mathrm{eff}}\right)$, representing the sum of the true surface gravity $g=G M / r^{2}$ and any other forces $\ddot{r}$ acting on the stellar photosphere,

$g_{\mathrm{eff}}=g+\ddot{r}$.

The final results for $T_{\text {eff }}$ and $\log g_{\text {eff }}$ are shown in Table 1 and in Fig. 2. The results for $v \sin i$ are also shown in Fig. 2. The behaviour of $g_{\mathrm{eff}}$ and $g$ as a function of $T_{\mathrm{eff}}$ through the pulsation cycle are compared with previous
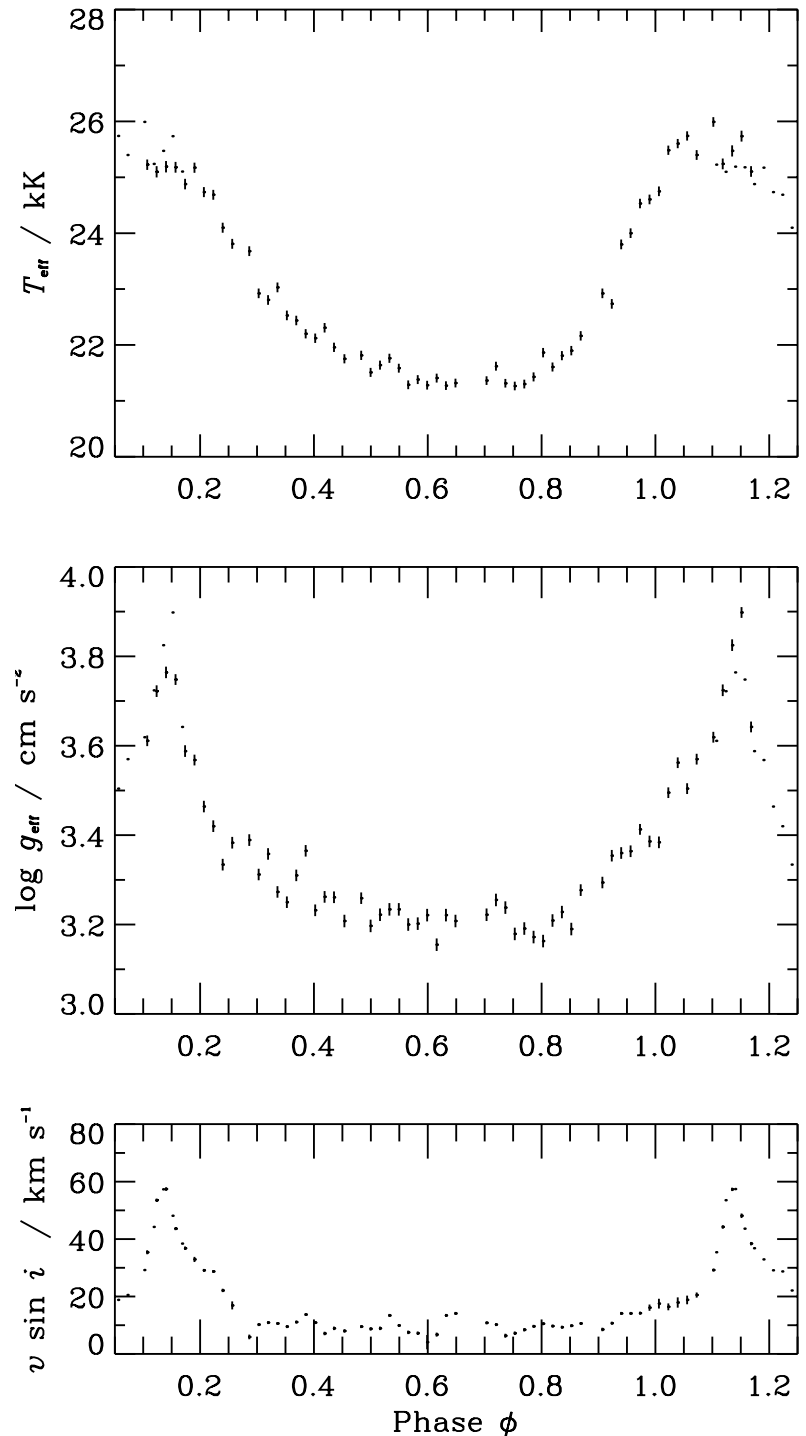

Fig. 2. The run of $T_{\text {eff }}, \log g_{\text {eff }}$ and $v \sin i$ as a function of phase as derived from the high-resolution spectra. The individual data for $T_{\text {eff }}$ and $\log g_{\text {eff }}$ are given in Table 1 . The values of $v \sin i$ represent the formal solution from the free-parameter fit; the excess broadening around minimum radius $(\phi \sim 0.15)$ is dicussed in the text. Vertical bars represent the formal measurement errors. The data are also shown folded by \pm 1 cycles (dots) to show the phase overlap.

measurements for the average values of these quantities in Fig. 3.

The robustness of these measurements was checked by removing a small number of lines, including He II $\lambda 4686 \AA$ A, from the fit. The change in $T_{\text {eff }}$ was less than $100 \mathrm{~K}$.

It is expected that $v \sin i$ should be approximately constant for a given star, although conservation of angular momentum may provoke a small increase in angular velocity as a pulsating star approaches minimum radius. It might seem logical, therefore, to adopt a constant value of $v \sin i$ for all spectra and treat any variation in other broadening mechanisms separately. However, the additional broadening profiles are not known a priori, while solving for " $v \sin i$ " explicitly makes an intrinsic and 


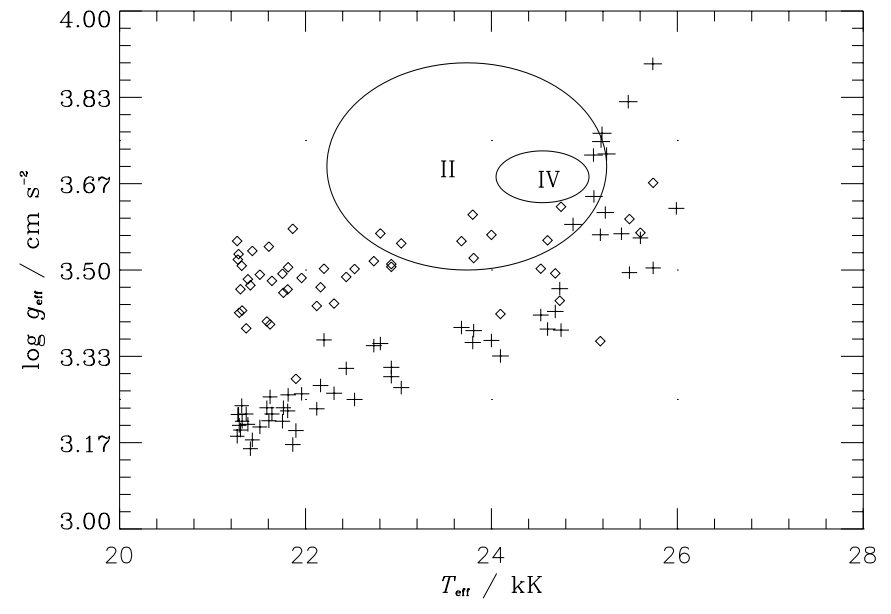

Fig. 3. The run of $T_{\text {eff }}$ versus $\log g_{\text {eff }}$ (with error bars) and $\log g(\diamond)$. The model grid used by SFIT is represented by dots. The loci of previous results for V652 Her are shown as II (Paper II: an ellipse indicating the total range of $T_{\text {eff }}$ and the error in $\log g$ ), and IV (Paper IV: an error ellipse showing the location of the phase-averaged spectrum).

necessary allowance for variations or errors in other parameters which affect the apparent linewidths.

Thus, around maximum radius $(0.3 \leq \phi \leq 0.9) v \sin i$ remains steady at $\sim 9 \pm 2 \mathrm{kms}^{-1}$, dependent upon the adopted $v_{\mathrm{t}}=9 \mathrm{~km} \mathrm{~s}^{-1}$. However, $v \sin i$ around minimum radius was considerably higher than this. On inspection, the spectral lines at phases $0.10 \lesssim \phi \lesssim 0.20$ are systematically broader than at other phases (Fig. 4). This does not appear to be due to projection broadening, the latter is a continuous function of expansion/contraction velocity and not acceleration. The observed effect far exceeds that of acceleration broadening, which was never greater than $10 \mathrm{kms}^{-1}$ for a $100 \mathrm{~s}$ exposure. Having considered every other possible source of line broadening in the current model, it is conjectured that dynamical processes at these phases violate the equilibrium assumption. There is evidence, for example, of doubling in some lines as might indicate the passage of a shock wave. This will be discussed further in Sect. 7. The failure of the models to correctly measure $v \sin i$ is reflected by the negative values for $g$ indicated in Table 1 . Around minimum radius, $\log g_{\text {eff }}$ is presumably underestimated in comparison with $\ddot{r}$ as a consequence of the breakdown of one or more equilibrium conditions assumed in the model atmosphere.

\section{3. $v_{t}$ and abundances}

In orded to measure the chemical composition in the atmosphere of V652 Her, SFIT_SYNTH was applied to five spectra close to maximum radius (run numbers 510, 512, 514, 516 and 518, Table 1) and also an average spectrum formed from run numbers 510-519. The wavelength scales of all spectra had been transformed into the laboratory reference frame prior to analysis. Near maximum radius, $T_{\text {eff }}$ and $\log g$ vary sufficiently slowly that forming such an average spectrum introduces negligible systematic errors. It does give a factor of three improvement in signal-to-noise ratio.

The value for microturbulent velocity, $v_{\mathrm{t}}$, was obtained by allowing it to vary at the same time as either the oxygen or the nitrogen abundance. Working with the average spectrum, values of 8.7 and $10.3 \mathrm{~km} \mathrm{~s}^{-1}$ were obtained from the nitrogen and oxygen lines respectively. Previously, $v_{\mathrm{t}}=5 \mathrm{~km} \mathrm{~s}^{-1}$ was obtained from O II lines alone (Paper IV), but this was from an average spectrum obtained around the pulsation cycle, including phases around minimum radius. The higher values are more consistent with measurements of $v_{\mathrm{t}}$ in other helium star atmospheres. There is virtually no difference between abundances derived with either 8.7 or $10.3 \mathrm{~km} \mathrm{~s}^{-1}$ and we adopt $9 \mathrm{~km} \mathrm{~s}^{-1}$ in the current analysis.

The abundances given by SFIT_SYNTH are shown in Table 2, where they are compared with the results obtained previously for V652 Her (Paper IV) and for related hydrogen-deficient stars (Drilling et al. 1998; Harrison \& Jeffery 1997; Jeffery \& Harrison 1997). There are reductions of up to a factor of two in the abundances of most species, primarily as a result of using a higher value for the microturbulence.

While all changes are within $3 \bar{\sigma}$ of the previous values, the previous error estimates for some individual species appear to have been optimistic, possibly as a consequence of adopting formal values, but probably because they do not include the systematic effect of $v_{t}$. The formal errors in the abundances given by SFIT_SYNTH are unrepresentatively small, typically 0.04 dex, although they do represent the spectrum-to-spectrum variation very well. They do not take into account errors in the atomic data, and systematic errors due to the adopted model atmospheres, $v_{\mathrm{t}}, T_{\text {eff }}$, etc.

An example of the quality of the fit between theoretical and observed spectrum is shown in Fig. 5. The astrophysical significance of the peculiar surface composition of V652 Her, which consists almost entirely of helium produced by the CNO-process, has already been discussed at length (Paper IV).

The measurement for phosphorus is unreliable. While our synthesis comprises some 17 phosphorus lines in the observed wavelength range, only two lines ( P II $\lambda 4499.2$ and $4589.9 \AA$ ) are clearly resolved. Contributions from very weak lines and lines in blends is likely to be responsible for skewing the abundance measured by SFIT_SYNTH. Given either the adopted abundance or one tenth of this value, both lines are too strong in the model compared with the observation (Fig. 5). The older value, based on stronger lines, should be preferred for the present. An alternative measurement of the phosphorous abundance could be obtained by removing weaker lines from the linelist input to SFIT_SYNTH. We remain sufficiently uncertain of the correctness of our models that this seems premature when a better result should be obtained from lines further in the blue. 


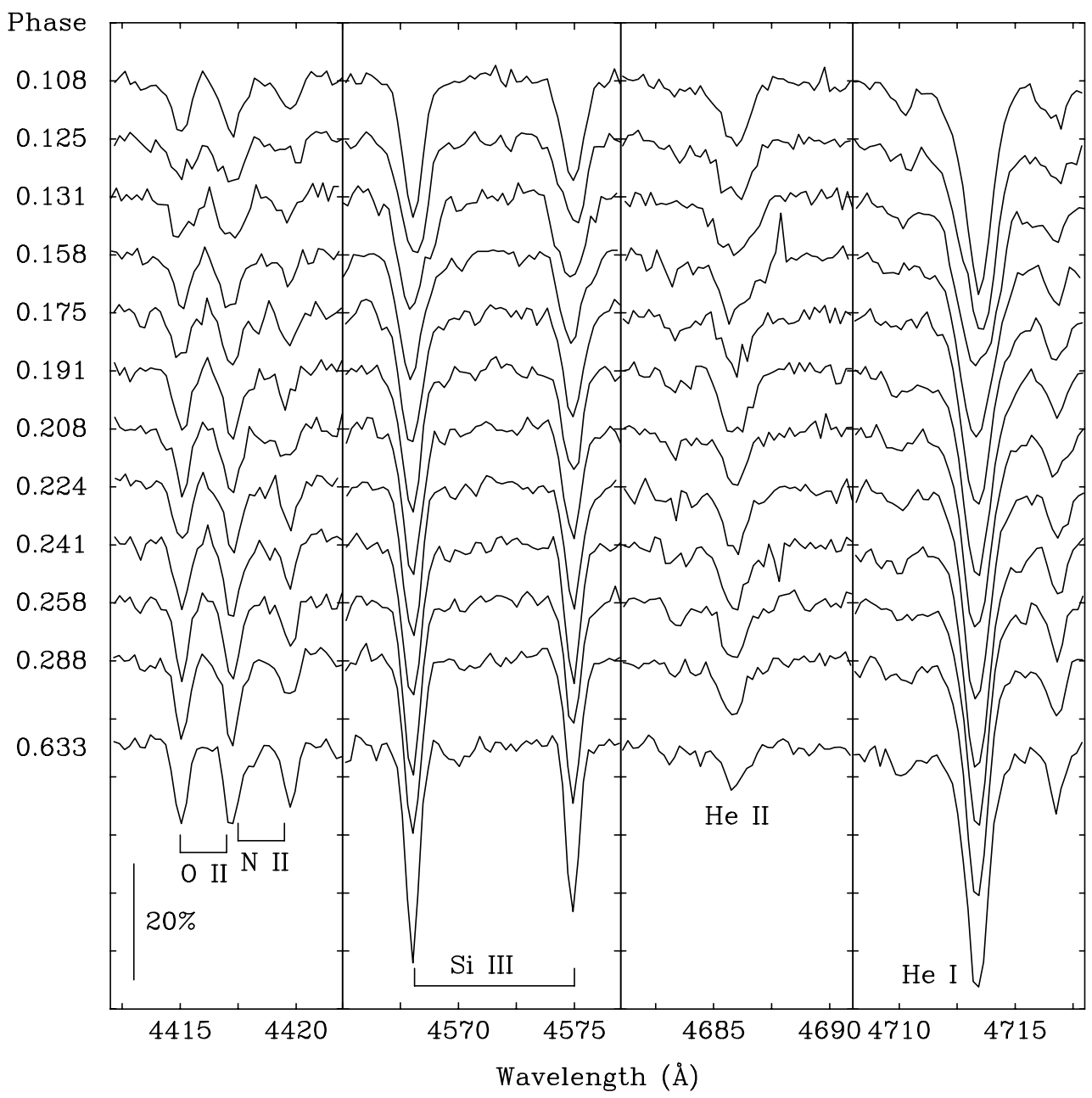

Fig. 4. A sequence of line profiles demonstrates line broadening around minimum radius. The pulsation phase for each spectrum is shown on the left hand side. A single spectrum obtained around maximum radius $(\phi=0.633)$ is shown for comparsion. Line profiles are shown for SiIII, He I, He II and other ions. The bar in the left hand panel representing $20 \%$ of continuum indicates the vertical scale. The wavelength scale is the same in all panels; tickmarks are separated by $2.5 \AA$. Wavelengths have not been transformed, so the rapid acceleration around minimum radius is visible as a change from a red to a blue shift. Note the behaviour in the line core of He I $\lambda 4713 \AA$, where the line core around phases 0.125 and 0.131 becomes significantly broader. This behaviour is exactly mimicked by He I $\lambda 4437 \AA$ (not shown).

Table 2. Atmospheric abundances of V652 Her, two similar gravity helium stars and the Sun. Abundances are given as log $n$, normalised to $\log \Sigma \mu n=12.15$.

\begin{tabular}{|l|r|r|r|r|r|r|r|r|r|r|r|r|r|r|}
\hline Star & $\mathrm{H}$ & $\mathrm{He}$ & $\mathrm{C}$ & $\mathrm{N}$ & $\mathrm{O}$ & $\mathrm{Ne}$ & $\mathrm{Mg}$ & $\mathrm{Al}$ & $\mathrm{Si}$ & $\mathrm{P}$ & $\mathrm{S}$ & $\mathrm{A}$ & $\mathrm{Fe}$ & $\mathrm{Ref}$. \\
\hline & $\log n$ & & & & & & & & & & & & & \\
V652 Her & 9.61 & 11.54 & 7.29 & 8.69 & 7.58 & 7.95 & 7.80 & 6.12 & 7.47 & 6.42 & 7.05 & 6.64 & 7.04 & \\
$(1999)$ & 9.38 & 11.54 & $7.14:$ & 8.93 & 7.54 & $8.38:$ & 7.76 & 6.49 & 7.49 & 5.35 & 7.44 & 6.73 & 7.40 & 1 \\
$\sigma$ & 0.07 & & $0.27:$ & 0.06 & 0.08 & $0.40:$ & 0.36 & 0.19 & 0.21 & 0.22 & 0.11 & 0.19 & 0.15 & \\
BX Cir & 8.1 & 11.5 & 9.02 & 8.4 & 8.0 & & & 7.2 & 6.0 & 6.8 & 5.0 & 6.6 & 6.6 & 2 \\
HD144941 & 10.3 & 11.5 & 6.80 & 6.5 & 7.0 & & & 6.1 & 4.8 & 6.0 & & & 5.7 & 3 \\
Sun & 12.0 & 11.0 & 8.55 & 7.97 & 8.87 & 8.08 & 7.58 & 6.47 & 7.55 & 5.45 & 7.23 & 6.56 & 7.50 & 4 \\
\hline
\end{tabular}

Notes: value uncertain.

References. (1) Paper IV; (2) Drilling et al. (1998); (3) Harrison \& Jeffery (1997); Jeffery \& Harrison (1997); (4) Grevesse et al. (1996). 

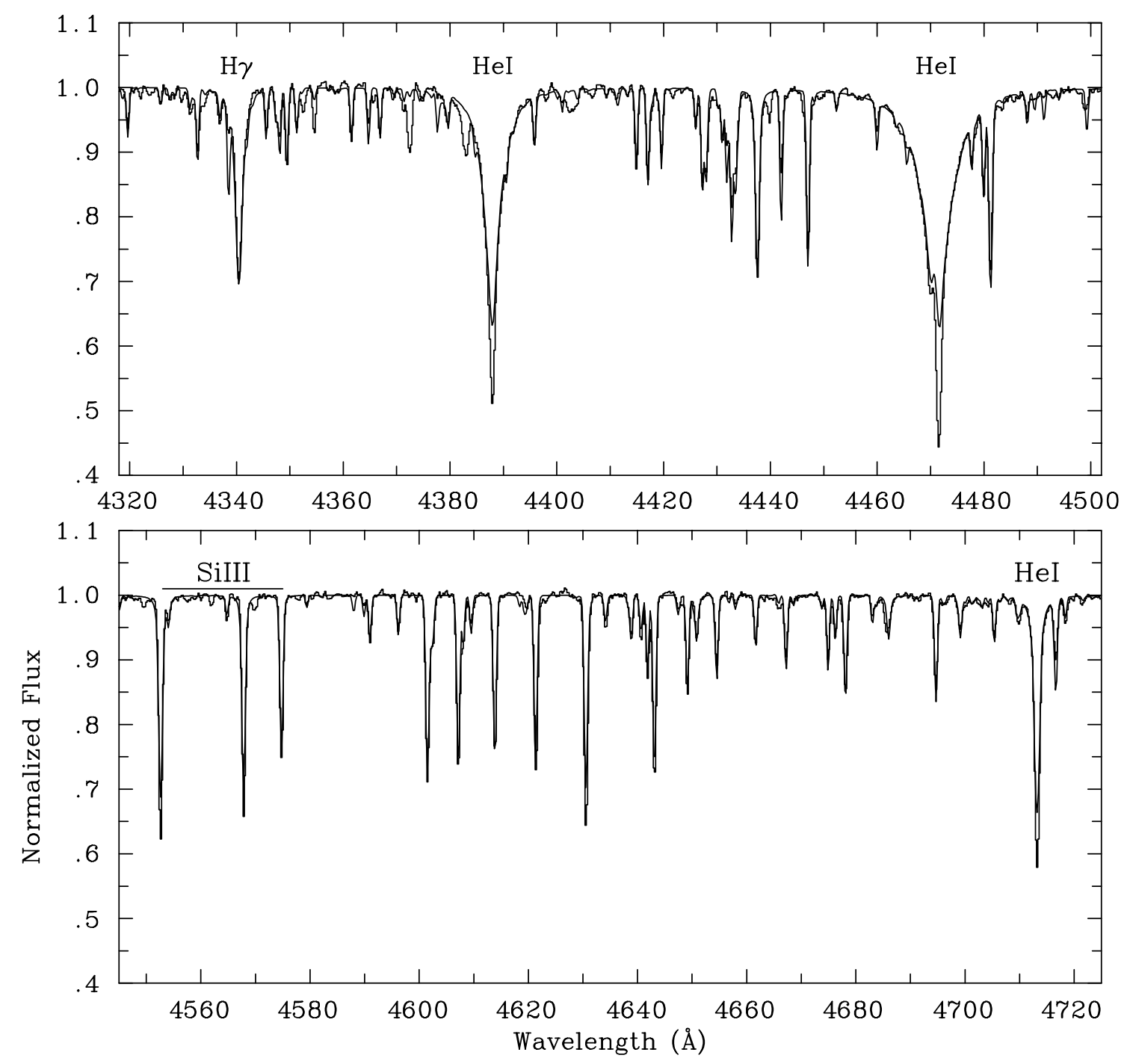

Fig. 5. Sections of the average normalized spectrum of the pulsating helium star V652 Her near maximum radius are shown (histogram) together with the synthetic spectrum (smooth curve) calculated using $T_{\text {eff }}=22000 \mathrm{~K}, \log g_{\text {eff }}=3.25(\operatorname{cgs}), v_{\mathrm{t}}=$ $9 \mathrm{kms}^{-1}, v \sin i=7 \mathrm{~km} \mathrm{~s}^{-1}$ and abundances given in Table 2. Selected strong lines have been labelled.

Table 3. IUE spectra and optical photometry used and temperatures and angular radii found in phase bins for V652 Her, assuming $E_{B-V}=0.06$.

\begin{tabular}{|c|c|c|c|c|c|c|c|c|c|c|c|c|}
\hline $\begin{array}{c}\text { phase } \\
\text { bin }\end{array}$ & $\begin{array}{c}\text { SWP } \\
\text { spectrum }\end{array}$ & $\begin{array}{l}\text { SWP } \\
\text { phase }\end{array}$ & $\begin{array}{c}\text { LWR } \\
\text { spectrum }\end{array}$ & $\begin{array}{l}\text { LWR } \\
\text { phase }\end{array}$ & $u$ & $v$ & $b$ & $V$ & \multicolumn{2}{|c|}{$\begin{array}{c}T_{\text {eff }} \pm \\
(\mathrm{kK})\end{array}$} & \multicolumn{2}{|c|}{$\begin{array}{cc}\theta & \pm \\
\left(10^{-11} \mathrm{rad}\right)\end{array}$} \\
\hline 0.11 & 09451 & 0.1274 & 15921 & 0.1029 & 10.421 & 10.502 & 10.494 & 10.558 & 22.15 & 0.25 & 2.93 & 0.014 \\
\hline 0.31 & 09444 & 0.3263 & 04883 & 0.3011 & 10.450 & 10.514 & 10.502 & 10.561 & 21.24 & 0.23 & 3.02 & 0.014 \\
\hline 0.38 & 05633 & 0.3724 & 15922 & 0.3877 & 10.457 & 10.507 & 10.493 & 10.551 & 20.81 & 0.21 & 3.08 & 0.014 \\
\hline & 09448 & 0.3970 & & & & & & & & & & \\
\hline 0.50 & 09453 & 0.5305 & 15926 & 0.4770 & 10.471 & 10.508 & 10.493 & 10.551 & 20.40 & 0.20 & 3.13 & 0.015 \\
\hline 0.59 & 09449 & 0.6180 & 15919 & 0.5730 & 10.487 & 10.516 & 10.501 & 10.559 & 20.08 & 0.19 & 3.16 & 0.016 \\
\hline 0.74 & 09445 & 0.7373 & 15927 & 0.7531 & 10.490 & 10.520 & 10.505 & 10.563 & 20.04 & 0.19 & 3.16 & 0.015 \\
\hline 0.80 & 09454 & 0.7914 & 15920 & 0.8187 & 10.485 & 10.518 & 10.503 & 10.561 & 20.41 & 0.20 & 3.11 & 0.015 \\
\hline 0.95 & 05799 & 0.9467 & 15924 & 0.9526 & 10.400 & 10.450 & 10.460 & 10.520 & 21.42 & 0.22 & 3.07 & 0.014 \\
\hline & 09446 & 0.9500 & & & & & & & & & & \\
\hline
\end{tabular}




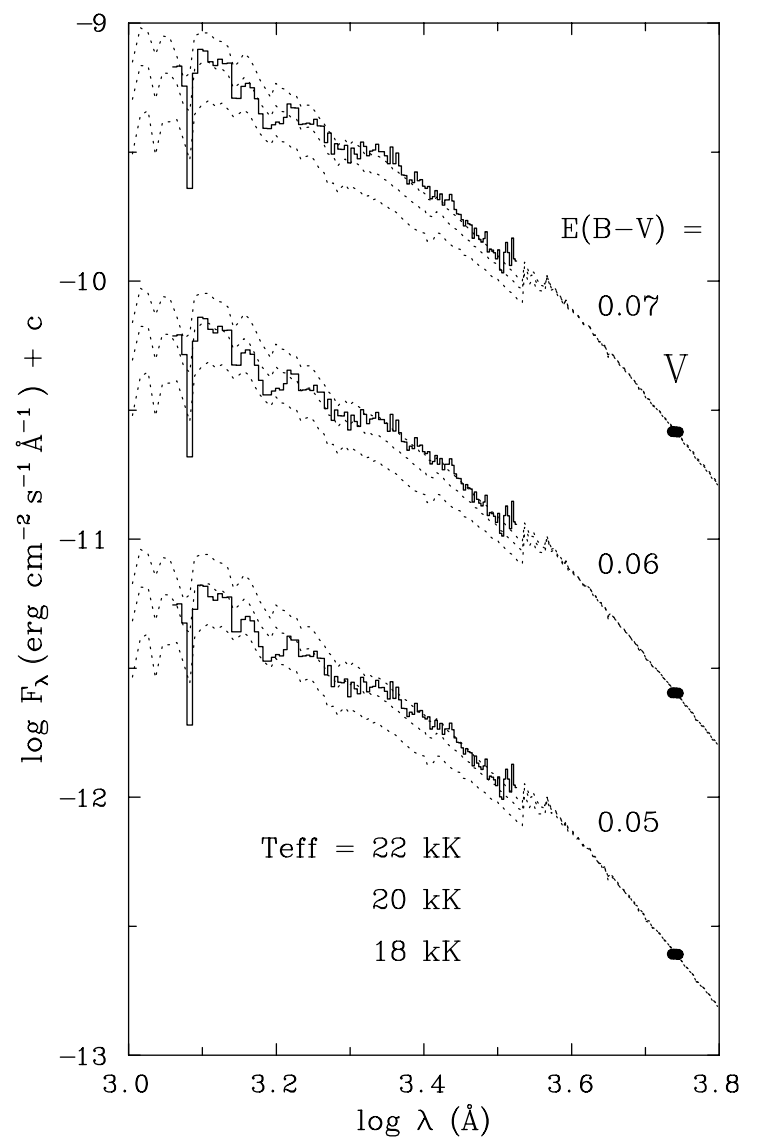

Fig. 6. IUE and visual spectrophotometry of V652 Her near maximum radius $(\phi=0.59$, bold histogram), with a series of model flux distributions, $T_{\text {eff }}=18,20,22 \mathrm{kK}$ (dotted lines). The observed fluxes have been binned by $20 \AA$ and dereddened by the amounts shown. The models have been normalized to the observed $V$ magnitude. The best fit solution had $T_{\text {eff }}=$ $20.1 \mathrm{kK}, E_{B-V}=0.06$.

\section{4. $T_{\text {eff }}, \alpha, E_{B-V}$}

The grid of STERNE line-blanketed model atmospheres described above was used with the fitting program FFIT to measure effective temperatures $T_{\text {eff }}$, angular diameters $\theta$, and interstellar extinction $E_{B-V}$, for each of the combined IUE spectra and from the Strömgren photometry.

We found that the $\log g$ used in the model atmospheres makes a negligible difference in the temperature and angular radius found by FFIT, so in the end only the $\log g=3.25$ models, appropriate to most of the pulsation cycle, were used.

In the analysis of the IUE data we used the spectral regions $1229 \AA \leq \lambda \leq 3200 \AA$. These wavelength cutoffs were chosen to remove regions where interstellar Ly $\alpha$ (short wave) and noise (long wave) caused trouble in the fitting procedure.

We initally ran FFIT with $E_{B-V}$ as a free parameter and with different combinations of data: a) IUE fluxes only; b) IUE fluxes $+V$ magnitude; c) Strömgren photometry $+V$ magnitude, d) IUE fluxes + Strömgren photometry $+V$ magnitude. Due to a coincidence between strong metal-blocking in the stellar spectrum and the interstellar absorption band at $2175 \AA$, fits (a) and (b) were unable to establish the exinction reliably, $E_{B-V}=0$ gave the best fits. With the addition of Strömgren photometry and considering data obtained between phases 0.3 and 0.9 (i.e. away from minimum radius), fits (c) and (d) gave $E_{B-V}=0.061 \pm 0.001$ and $E_{B-V}=0.059 \pm 0.001$ respectively. We here adopt $E_{B-V}=0.06$.

This is slightly different to $E_{B-V}=0.07$ found in previous work (e.g. Paper II). The likely source of the difference is that our analysis includes line blanketing. In these analyses it can be difficult to distinguish between line blanketing in the star and interstellar extinction. Both effects tend to depress the observed flux around $2175 \AA$. Using line blanketing in the models means less extinction is needed to match the synthetic spectra to the observations. Fits to the observed flux distribution near maximum radius are shown in Fig. 6.

The temperatures and angular radii found with the specified input data are shown in Table 3 and Fig. 13. We find the mean temperature through the pulsation cycle to be $20.0 \mathrm{kK}$. This value is lower by $3.0 \mathrm{kK}$ than that obtained from the ionization equilibrium in the optical spectroscopy.

A likely reason for the discrepancy is the treatment of line opacity $\left(\kappa_{1}\right)$ in the model atmospheres. The line lists used to construct the distribution functions were far from complete (see Appendix A.1) and tailored for extreme helium stars. Thus the hydrogen Lyman line opacity will have been underestimated by a factor $\sim 10$ and the carbon line opacity considerably overestimated. A comparison of the low-dispersion fluxes from the models (STERNE) with synthetic high-resolution optical spectra (SPECTRUM) indicates that $\kappa_{1}$ could be too low by a factor of as much as two. Increasing the total $\kappa_{1}$ would increase back-warming in the atmosphere, raising the temperature of the continuum forming layers relative to that of the line-forming layers.

As a simple experiment, a model atmosphere with $T_{\text {eff }}=22.0 \mathrm{kK}$ and $\log g=3.50$ was computed with $\kappa_{1}$ multiplied by two at all wavelengths. The theoretical ultraviolet spectral energy distribution and the highresolution otical spectrum predicted by this model were compared with the standard models using FFIT and SFIT. The experimental energy distribution was measured to have $T_{\text {eff }}=20.5 \mathrm{kK}$ and the high-resolution spectrum gave $T_{\text {eff }}=21.8 \mathrm{kK}, \log g=3.39$. Consequently, increasing $\kappa_{1}$ in the model grid would have had the opposite effect on the measurements for V652Her, increasing both $T_{\text {eff }}$ and $\log g$, and reducing the discrepancy between the two measurements of $T_{\text {eff }}$. It is clear that a more appropriate treatment of the line opacity is urgently required.

\subsection{Lyman $\alpha$ and $E_{B-V}$}

An independent measurement of the extinction can be obtained from the interstellar contribution to the 
hydrogen Ly $\alpha$ line profile. Two high-resolution observations of V652 Her were obtained with the SWP camera on IUE in 1983 (image numbers SWP19841 and SWP 19966). The reduced and calibrated images have been recovered from the INES data archive and an average spectrum has been constructed. The region around Ly $\alpha$ is shown in Fig. 7.

The predicted stellar contribution to Ly $\alpha$ is comparatively weak (dashed line in Fig. 7), and is obscured by geocoronal Ly $\alpha$ emission and the interstellar Ly $\alpha$ absorption profile. The latter can be obtained as a function of the hydrogen column $N(\mathrm{H})$ (Groenewegen \& Lamers 1989) and hence of the extinction $E_{B-V}$ (Bohlin et al. 1978). The upper-left panel in Fig. 7 shows the contributions of interstellar Ly $\alpha$ alone, and suggests that values of $E_{B-V}$ between 0.10 and 0.15 might be appropriate. However, it is necessary to consider the metal-line spectrum as well in order to establish the true Ly $\alpha$ profile - as is suggested by the apparently high continuum placement.

The continuum level has been estimated by constructing an experimental synthetic spectrum for V652 Her around Ly $\alpha$. We assumed a typical model atmosphere corresponding roughly to maximum radius and measured abundances from the optical analysis, except for that of silicon which was reduced by 1 dex in order to approximately match the profile of Si III $\lambda 1206.5 \AA$. Abundances of additional elements were assumed to be solar. A linelist comprising some 10000 lines between 1170 and $1260 \AA$ (Kurucz \& Petryemann 1975; Kurucz 1988; Hubeny et al. 1994) was compiled, but not critically evaluated. Therefore this spectrum is only statistically representative of line absorption in the region and only useful for estimating the continuum level and interstellar Ly $\alpha$ profile.

After convolution with an interstellar Ly $\alpha$ profile for $E_{B-V}=0.07$, the theoretical spectrum is found to be satisfactorily similar to the observed spectrum (Fig. 7: bottom panel). Allowing for some uncertainty in the metal abundances and errors in the construction and choice of model atmosphere, an error of \pm 0.02 in $E_{B-V}$ might be entertained, but fits with $E_{B-V}=0.05$ or 0.10 are demonstrably poorer than that adopted (Fig. 7: upper-right).

\section{Infrared spectroscopy}

Being the first infrared spectrum of an early-type helium star, the UKIRT spectrum (Fig. 8) is significant. Several HeI and two hydrogen Paschen lines are easily identified (Moore 1945). The hydrogen lines are considerably stronger than the He I lines, despite the latter being approximately 100 times more abundant. Most remarkably, the normally strong He $\lambda \lambda 10830 \AA$ line is undetectable. A difficulty with analysing such data is that strong infrared lines of hot stars are considered to be affected by departures from LTE. Nevertheless, an LTE model may demonstrate where attention should be focused.

SPECTRUM has been substantially extended by including Stark broadening data (electron damping widths) for infrared lines calculated from the impact approximation (Dimitrijevic \& Sahal-Brechot 1989; Dimitrijevic \& Sahal-Brechot 1990). These are tabulated as a function of electron density and temperature. Calculations were made using both constant values $(T=$ $\left.20000 \mathrm{~K}, \log n_{\mathrm{e}}=15\right)$ and interpolated values. A small difference in the line profiles was found for the diffuse lines, leading us to adopt the interpolated values.

The theoretical spectrum computed from a $T_{\text {eff }}=$ $22000, \log g=3.5$ model atmosphere with $n_{\mathrm{H}}=0.011$ (Table 2) is shown in Fig. 9. The predicted hydrogen lines are substantially weaker than observed - as expected but contrary to observation. The diffuse He I lines are the strongest lines, again as expected and broadly in agreement with observation. He I $\lambda 10830 \AA$ is predicted to be stronger than the neighbouring $3^{3} P-6^{3} S$ line, which can be detected in the UKIRT spectrum. Why it is not detected will require high-resolution observations of He I $\lambda 10830 \AA$ around the pulsation cycle and a consideration of how departures from LTE affect this line in particular.

\section{The radius of $\mathrm{V} 652 \mathrm{Her}$}

\subsection{Radial velocity transformation}

Direct methods for measuring the radii of pulsating stars require a transformation from the measured $(v)$ to the actual radial velocity $(\dot{r})$ of the stellar surface, and hence to the integrated displacement $(\delta r)$. The integration over the stellar disk of a spherical expansion projected into the line of sight has been examined at length during the last century (e.g. Shapley \& Nicholson 1919; Parsons 1972), but in general only for the case of Cepheids. The behaviour of the projection factor $p$ has only recently been examined for hydrogen-deficient early-type pulsators by Montañés Rodriguez \& Jeffery (2001a). They find that for radial velocities of V652 Her measured using the same crosscorrelation procedure as here (Sect. 3 ), $\dot{r}$ is given by:

$\dot{r}=-p(v-\bar{v})=-p \delta v$

if

$p=1.402-0.028 \gamma$,

where $\gamma=|\dot{r}| / w_{1 / 2}$ is the expansion velocity scaled by the width (in velocity units) at half depth of the line (wavelength $\lambda) w_{1 / 2}=\Delta \lambda_{1 / 2} c / \lambda$. The variation with velocity was not unexpected; for example Parsons (1972) showed that for isolated lines in Cepheids, $p$ depends on linewidth $\left(\Delta \lambda_{1 / 2} / \lambda_{0}\right)$ and shift $(\delta v)$

$p=1.37-\frac{0.039 \lambda_{0}}{c \Delta \lambda_{1 / 2}}|\delta v| \equiv 1.37-0.042 \gamma$.

It is important to note that an increase in $p$ results in a proportional increase in the radius measured directly from combined radial velocity and angular diameter variations. 

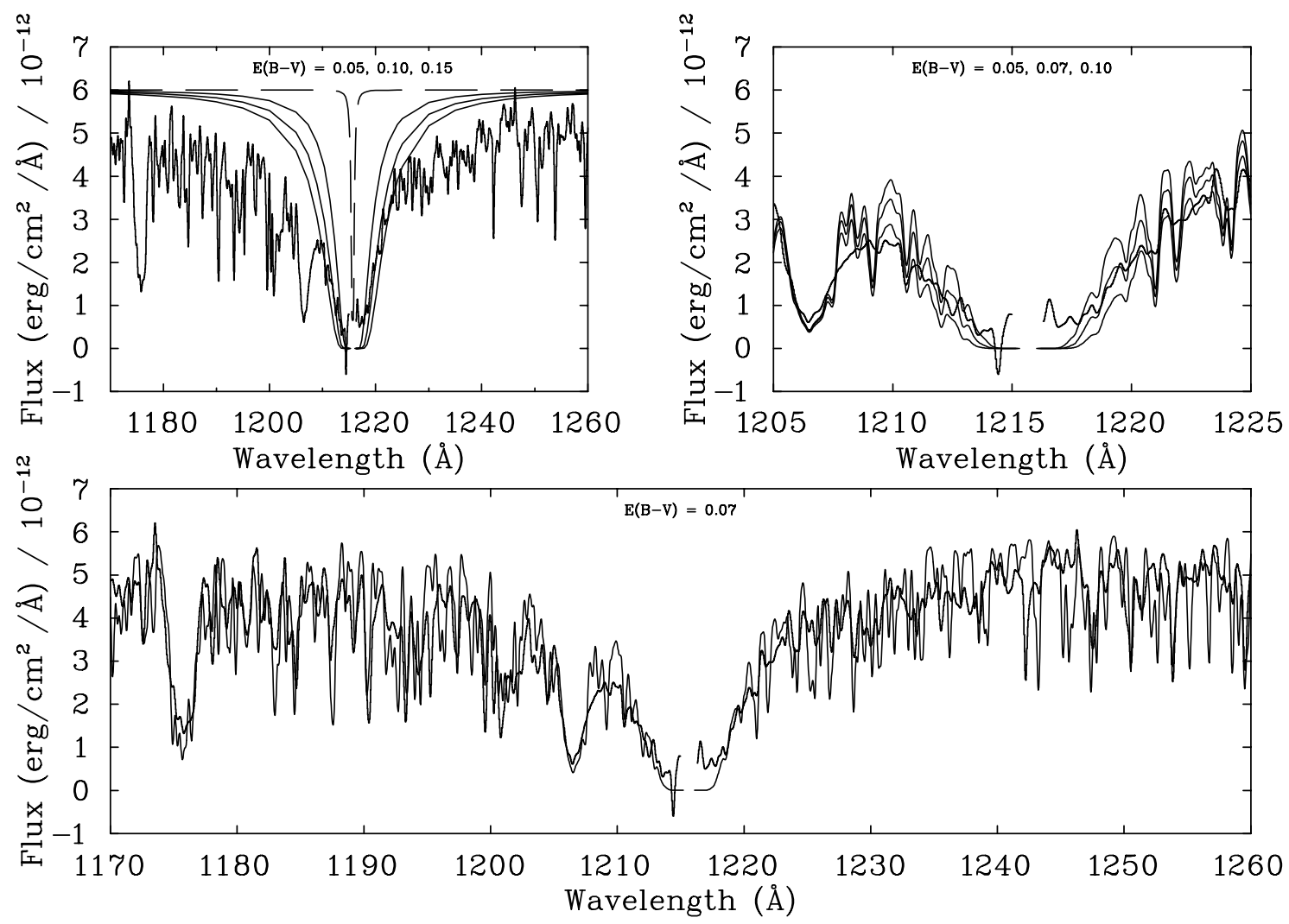

Fig. 7. IUE high-resolution spectrum of V652 Her around hydrogen Ly $\alpha$ convolved with a $0.2 \AA$ Gaussian (heavy line, all panels). Geocoronal emission has been removed. The predicted stellar contribution to Ly $\alpha$ (dashed line) convolved with interstellar absorption computed for $E_{B-V}=0.05,0.10$ and 0.15 is shown in the upper-left panel (thin lines). A synthetic spectrum for the whole region, convolved with interstellar absorption computed for $E_{B-V}=0.07$ only is shown in the lower panel, also convolved with a $0.2 \AA$ Gaussian (thin line). The upper-right panel shows the central $20 \AA$ of the Ly $\alpha$ profile compared with similar models for $E_{B-V}=0.05,0.07$ and 0.10 . The lowermost model corresponds to the largest value of $E_{B-V}$.

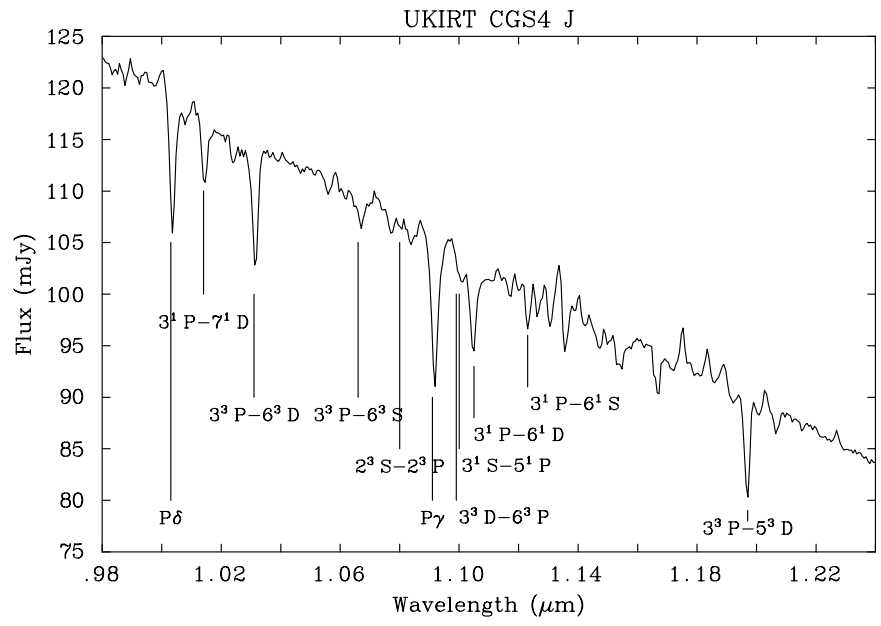

Fig. 8. CGS4 $J$-band spectrum of V652 Her showing He I (labelled by transition) and hydrogen Paschen lines $(\mathrm{P} \gamma, \delta)$.

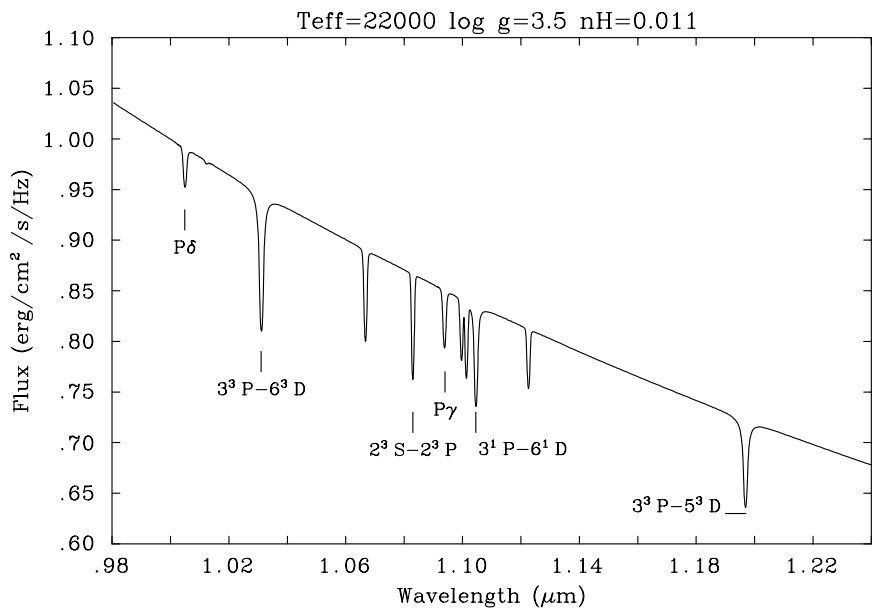

Fig. 9. LTE model spectrum for V652 Her matching the UKIRT $J$-band spectrum in Fig. 8. The model parameters are shown (top). He I $\lambda 10830 \AA$, diffuse He I lines and hydrogen Paschen lines $(\mathrm{P} \gamma, \delta)$ are also marked. The model fluxes have been normalized to unity at $1 \mu \mathrm{m}$ and smoothed with a $F W H M=0.6 \mathrm{~nm}$ Gaussian to emulate the instrumental resolution of the UKIRT spectrum. 

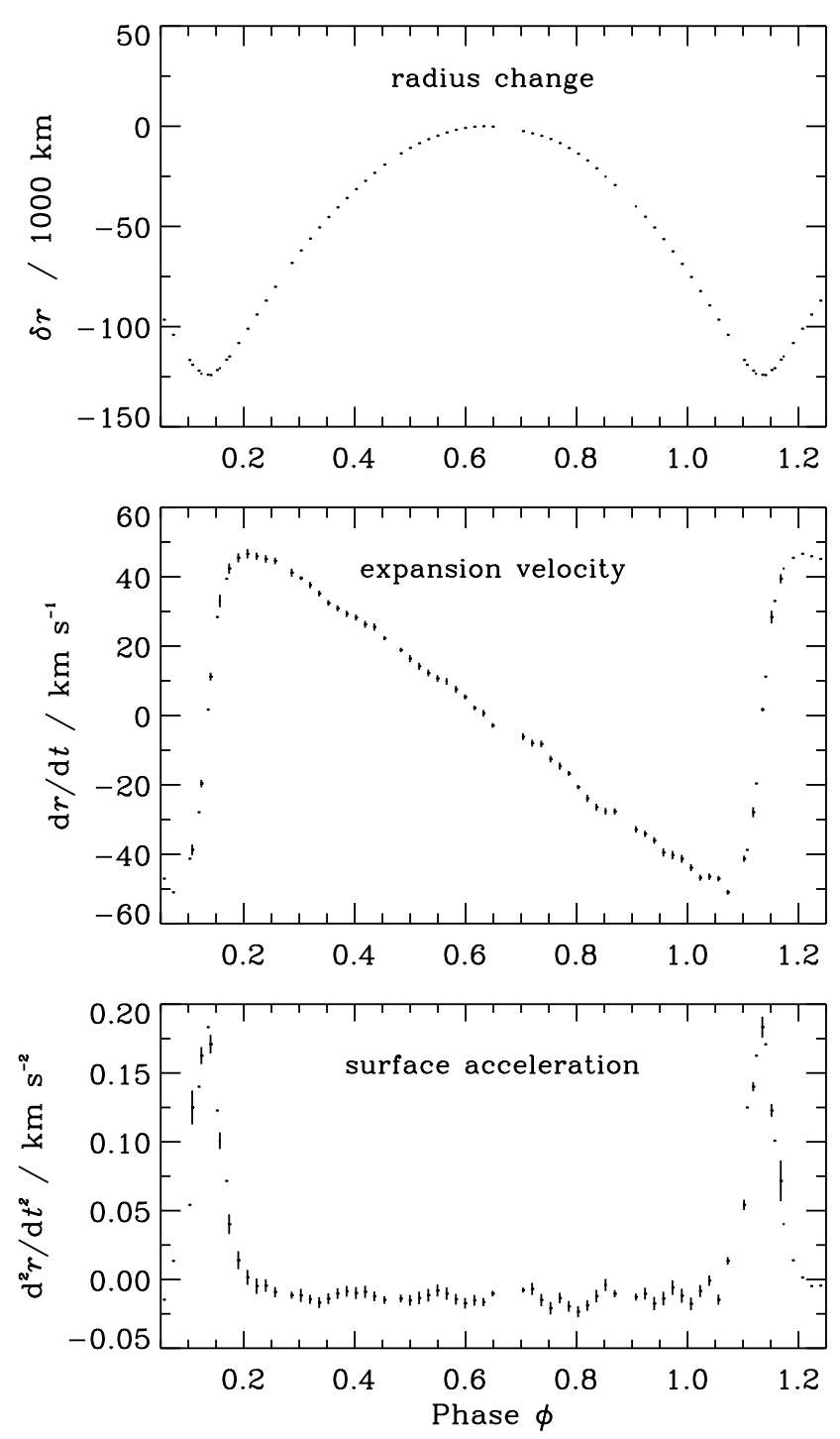

Fig. 10. The change in total radius ( $\delta r$ top), the expansion velocity $(\dot{r}$, center) and the surface acceleration $(\ddot{r}$, bottom) of $\mathrm{V} 652$ Her as a function of pulsation phase. The vertical bars represent the propagated measurement errors. The dots represent the same data folded over \pm 1 cycle.

Applying Eqs. (3) and (4) to the data for $v$ in Table 1 yields $\dot{r}$. The acceleration $\ddot{r}$ and radius change $\delta r$, defined as

$\ddot{r}_{i}=\frac{\dot{r}_{i+1}-\dot{r}_{i-1}}{t_{i+1}-t_{i-1}}$

and

$\delta r_{i}=\int_{t_{0}}^{t_{i}} \dot{r} \mathrm{~d} t$

are then derived; $t_{i}$ are the times of observation. The true surface gravity can then be derived from the apparent surface gravity using Eq. (2). The derived quantities $\dot{r}, \ddot{r}, \delta r$ and $g$ are given in Table 1.
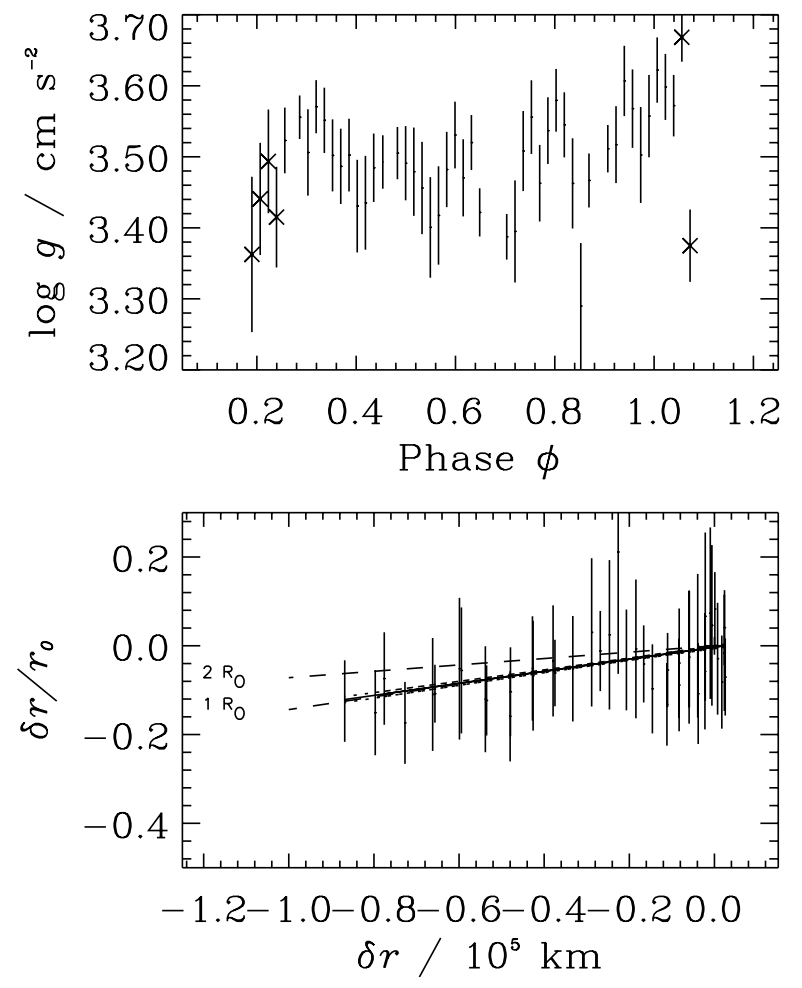

Fig. 11. The change in relative radius $\left(\sqrt{g_{0} / g}-1=\delta r / r_{0}\right)$ as given by the surface gravity method is plotted against the total change in radius $(\delta r$, lower panel). The total stellar radius corresponding to the reference phase $\left(r_{0}\right)$ is given by the inverse slope of the best fit line. Lines with gradients corresponding to 1 and $2 R_{\odot}$ are also shown (dashed). The corresponding true surface gravity $g$ obtained from Eq. (2) is shown in the upper panel. Measurements close to minimum radius and marked " $x$ " were omitted from the regression.

\subsection{Radius from surface gravity measurements}

Classical methods such as Baade's method and the BaadeWesselink method for measuring the radii of radially pulsating stars depend on photometry to provide the angular radius variation as a function of phase. A substantial volume of visual and ultraviolet photometric data for V652 Her is already available and has been used to good effect (Paper II). We have reanalyzed this data (Sect. 6.4), in order to ensure that our own methods are fully consistent with these earlier results. Further progress can no doubt be achieved with the addition of infrared photometry to further constrain the total flux measurement.

However, to supplement Baade's approach, we here propose and apply a novel method for measuring the angular radius variation in a radially pulsating star. The advantage of this method for us is that it is completely independent of any previous measurement or analysis and depends only upon observational material already presented in this paper. The disadvantage is that the result may not be so precise.

The key to this method is the precise measurement of surface gravity as a function of phase. The graph of displacement $\delta r=r-r_{0}$ against $\sqrt{g_{0} / g}-1 \equiv\left(r-r_{0}\right) / r_{0}$, 
Table 4. Dimensions of V652 Her using three different methods employing surface gravity $(g)$, visual magnitude $(V)$ and ultraviolet spectrophotometry (IUE). The results are presented in two groups, the first referring to the stellar dimension at maximum radius $\phi_{0}$, the second to the cycle-averaged dimensions. Previous results (Paper II) are shown for comparison.

\begin{tabular}{|l|rr|rr|rr|rr|}
\hline & $g$ & \pm & $V$ & \pm & IUE & \pm & Paper II & \pm \\
\hline$\phi_{0}$ & 0.626 & 0.005 & 0.626 & 0.005 & 0.59 & & $\dagger 0.856$ & \\
$T_{\text {eff }} / \mathrm{kK}$ & 21.42 & 0.16 & 21.42 & 0.16 & 20.08 & 0.19 & 23.20 & 1.40 \\
$\theta_{0} / 10^{-11} \mathrm{rad}$ & & & 3.10 & 0.02 & 3.16 & 0.02 & 2.97 & 0.10 \\
$\log g_{0} / \mathrm{cm} \mathrm{s}^{-2}$ & 3.46 & 0.05 & 3.46 & 0.05 & 3.46 & 0.06 & & \\
$R_{0} / R_{\odot}$ & 1.06 & 0.18 & 1.32 & 0.003 & 2.37 & 0.16 & & \\
$L_{0} / L_{\odot}$ & 211 & 50 & 331 & 5 & 821 & 80 & & \\
$M / M_{\odot}$ & 0.11 & 0.03 & 0.18 & 0.02 & 0.59 & 0.09 & & \\
$d /$ parsec & & & 963 & 6 & 1693 & 115 & & \\
$E_{B-V}$ & & & & & & & & \\
$<T_{\text {eff }}>/ \mathrm{kK}$ & 22.93 & 0.01 & 22.93 & 0.01 & 20.95 & 0.07 & 23.45 & 1.32 \\
$<\theta>/ 10^{-11} \mathrm{rad}$ & & & 2.97 & 0.00 & 3.07 & 0.01 & & \\
$<\log g>/ \mathrm{cm} \mathrm{s}{ }^{-2}$ & 3.50 & 0.06 & 3.48 & 0.12 & 3.48 & 0.12 & 3.7 & 0.2 \\
$<R>/ R_{\odot}$ & 0.99 & 0.02 & 1.26 & 0.00 & 2.31 & 0.02 & 1.98 & 0.21 \\
$<L>/ L_{\odot}$ & 244 & 8.4 & 393 & 1 & 919 & 14 & 1070 & 340 \\
$<M>/ M_{\odot}$ & 0.11 & 0.02 & 0.17 & 0.05 & 0.59 & 0.18 & 0.7 & ${ }_{-0.3}^{+0.4}$ \\
$<d>/$ parsec & & & 957 & 1 & 1695 & 16 & 1500 & 100 \\
\hline
\end{tabular}

$\dagger$ Reference phase (RP).

where the subscript 0 refers to a reference phase such as maximum radius, should then be a straight line with gradient $r_{0}$.

The surface gravity method has been applied to the data of Table 1. Data points with $0.05<\phi<0.30$ (around minimum radius) have been excluded because the gravity measurement appears to become unreliable as the equilibrium assumption breaks down.

As reference phase, we have adopted $\phi_{0}=0.626 \pm 0.027$ representing the mean phase of runs $512-525$. Values for $r_{0}, g_{0}$ and $\alpha_{0}$ are thus also defined at this phase, so that $\log g_{0}=3.46 \pm 0.05$. The regression shown in Fig. 11 gives $r_{0}=1.06 \pm 0.18 R_{\odot}$.

Even with the quality of the present data, this method measures the radius to no better than 20 per cent and possibly worse. It is susceptible to errors in measuring the He I line profiles, to systematic errors in the model atmopsheres and in the atomic data for HeI. We cannot therefore rely on this measurement alone.

\subsection{Radius from spectroscopic temperature and visual magnitudes}

Since we have measured the effective temperature spectroscopically, an alternative approach is to measure the angular radius using the visual light curve of Kilkenny \& Lynas-Gray (1982, Fig. 12). This is achieved by normalising the $V$-band flux from a model atmosphere with effective temperature $T_{\text {eff }}(\phi)$ to the observed visual magnitude $V(\phi)$. The resulting angular radius $\theta(\phi)$ (Fig. 12) can then be used, as before to define the radius $\theta_{0}$ at reference phase $\phi_{0}$. The stellar radius is obtained from

$\frac{\left(\theta-\theta_{0}\right)}{\theta_{0}} \equiv \frac{\left(r-r_{0}\right)}{r_{0}}=\frac{\delta r}{r_{0}}$

where $\theta, \theta_{0}$ and $\delta r$ are known. The regression shown in Fig. 12 gives $r_{0}=1.325 \pm 0.003 R_{\odot}$, in reasonable agreement with the result obtained from the $\log g$ measurement. This method assumes that the $V$ magnitude and spectroscopic $T_{\text {eff }}$ may be combined to derive the angular diameter. If the model atmospheres are correct, this should be true.

\subsection{Radius from ultraviolet and optical spectrophotometry}

The angular radii found with FFIT were used with the changes in radius found from radial velocity measurements (Sect. 3) to plot $\delta \theta / \theta_{0}$ versus $\delta r$ (Fig. 13). If all data are used (eight points), the regression gives $r_{0}=$ $2.37 \pm 0.16 R_{\odot}$, where the uncertainty is derived from the standard error in the least squares fit to the slope. The result is practically identical to an earlier analysis of the same data (Paper II), given that the current $r_{0}$ and the previous $\langle R>$ are defined slightly differently.

If $E_{\mathrm{B}-\mathrm{V}}=0.07$ is chosen, as in previous analyses, instead of 0.06 , then the estimated mean temperature 

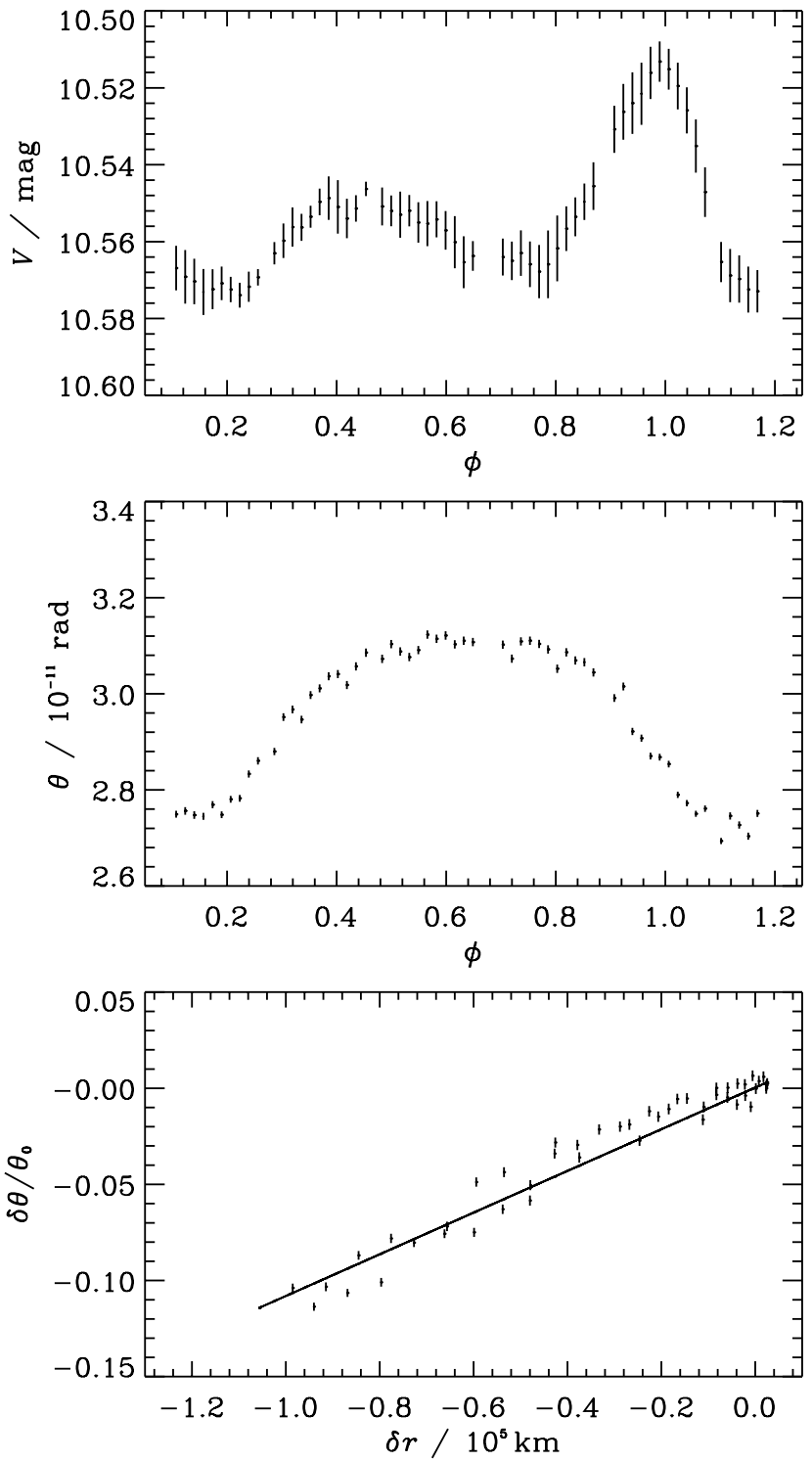

Fig. 12. Derivation of radius from visual photometry (Kilkenny \& Lynas-Gray 1982, top panel). The angular radius $\theta$ is estimated by fitting model flux distributions corresponding to the spectroscopic $T_{\text {eff }}$ to the visual photometry (centre). The radius may be deduced from the gradient of $\delta \theta / \theta_{0}$ with $\delta r$ (bottom panel).

increases but the derived radius is almost unaffected. Tests run with several extinction values between 0.03 and 0.15 did not change the estimated stellar radius by more than $0.02 R_{\odot}$. The variations were uncorrelated with the extinction used.

There is a clear difference between the measurements of $r_{0}$ obtained from optical data alone and that derived here. A partial contribution to the discrepancy may be seen in Fig. 13 where one IUE datum lies close to minimum radius while the remainder lie within \pm 0.3 cycles of maximum radius. Hence the $\delta r$ volume is not well sampled compared with the optical spectroscopy. If the stellar photosphere at $\phi \sim 0.1$ is out of LTE, this single datum would seriously alias the result. However, omitting it only reduces the value of $r_{0}$ to $2.2 \pm 0.4 R_{\odot}$.
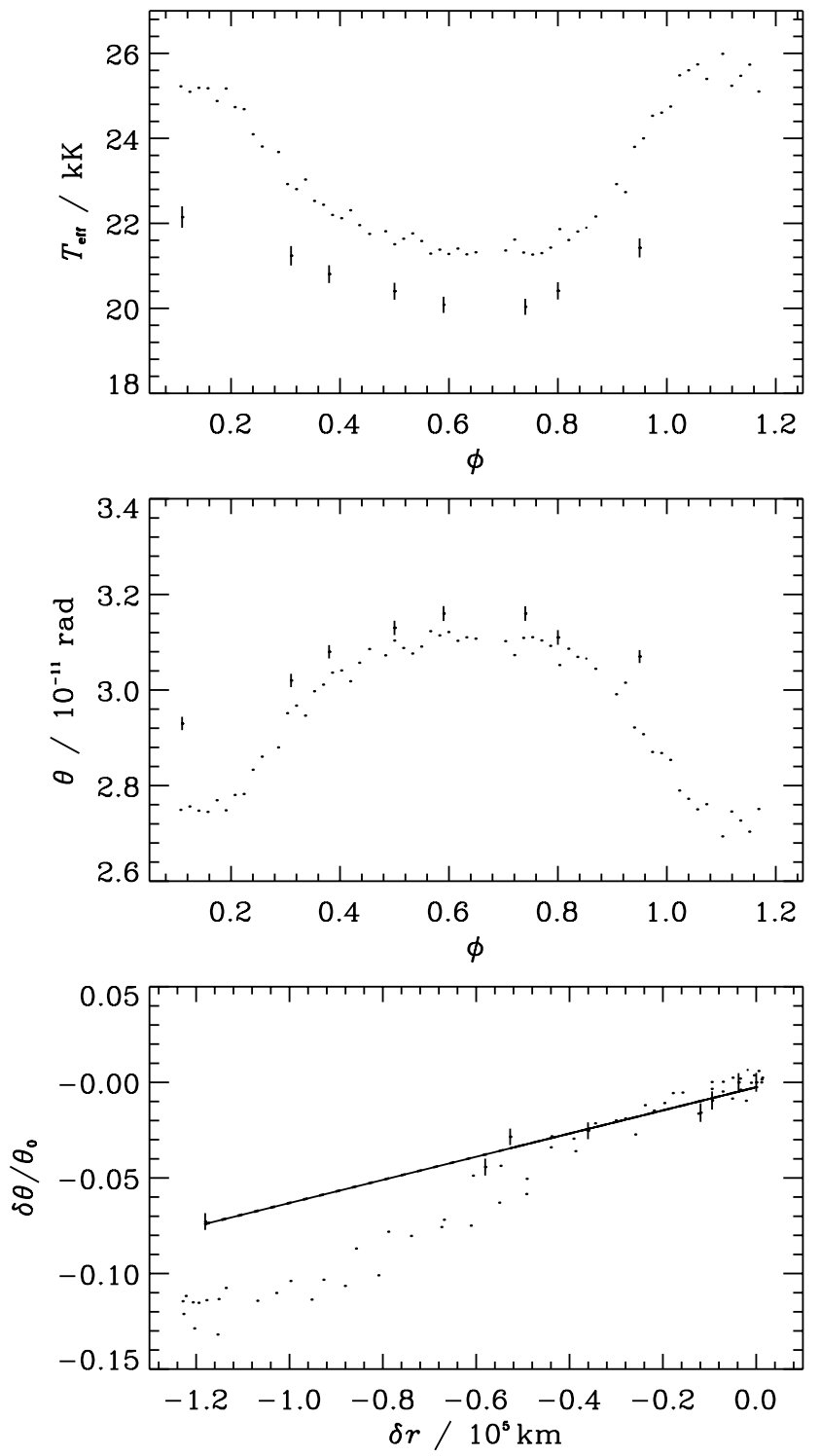

Fig. 13. Derivation of radius from ultraviolet and visual spectrophotometry. The effective temperature $T_{\text {eff }}$ (top) and angular radius $\theta$ (center) obtained by fitting model flux distributions to combined IUE spectrograms and appropriate data from the visual light curve (Kilkenny \& Lynas-Gray 1982) are shown in Table 3 . The values of $\theta$ obtained from the visual photometry and spectroscopic temperatures (Fig. 12) are overplotted (dots). The stellar radius may be deduced from the gradient of $\delta \theta / \theta_{0}$ with $\delta r$ (bottom).

\subsection{Mass, luminosity and distance}

Having measured $g_{0}, \theta_{0}, r_{0}$ and $T_{\text {eff } 0}$, the values of other stellar dimensions including mass $M$, luminosity $L$ and distance $d$ follow from standard identities. Since these refer in general to the stellar dimensions at the reference phase (maximum radius, subscript 0 ), they may be adjusted by integration around the pulsation cycle to give average values for $\left\langle T_{\text {eff }}\right\rangle,\langle\log g\rangle,\langle r\rangle$ and $\langle L>$. The values of $M$ and $d$ obtained from reference phase and cycle-average values are, as expected, the same to within measurement errors. All of these values are given in Table 4. 


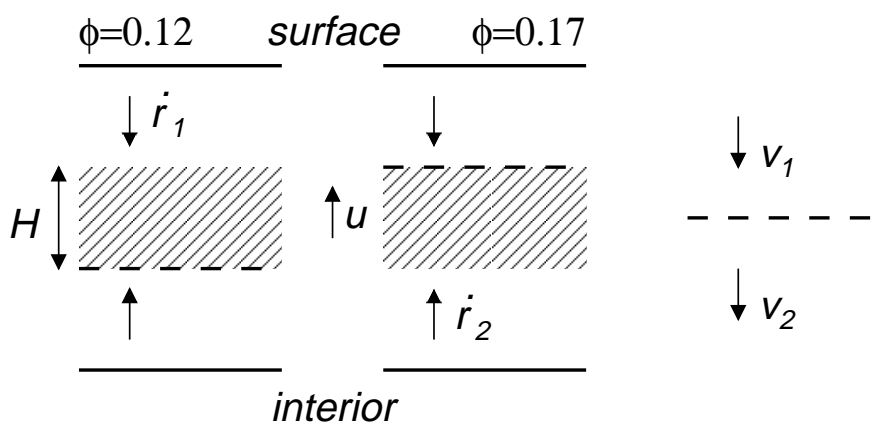

Fig. 14. Schematic of the compression wave (dashed line) passing through the line-forming region (shaded area) in the stellar rest frame (left) and in the compression-wave rest frame (right).

V652 Her was observed by Hipparcos (Schrijver 1997) but the measured parallax ( $p=0.90 \pm 1.77$ mas) is unhelpful although quite consistent with the distances given in Table 4.

It is appropriate to comment on the difference in $r$ derived from optical spectroscopy and visual photometry and that derived after including ultraviolet spectrophotometry. In the first case, optical spectroscopy fixes $T_{\text {eff }}$ and the $V$-band photometry fixes the angular radius $\theta$; the total flux from the star is not considered. In the second case, the ultraviolet fluxes establish $T_{\text {eff }}$, Since the measurement of $r$ depends on $\delta \theta / \theta$, scaling errors should not be important providing $\mathrm{d} \theta / \mathrm{d} T_{\text {eff }}$ is constant. However, this quantity is $\sim 30 \%$ larger for the ultraviolet fluxes than for the optical spectroscopy which results in $r$ being larger by a similar amount. The difference in $\mathrm{d} \theta / \mathrm{d} T_{\text {eff }}$ must be a consequence of systematic errors in the model atmospheres; inadequate line opacities have already been discussed (Sect. 4.4), departures from equilibrium will be discussed below.

\section{Hydrostatic equilibrium}

It has been shown that the surface acceleration of V652 Her at minimum radius is large $\left(\sim 0.17 \mathrm{~km} \mathrm{~s}^{-2}\right)$ and that the absorption lines are broader than indicated by the hydrostatic approximation. The question of whether the latter is a valid approximation for spectral analysis has already been introduced. With the results derived above, it is possible to examine the question in more detail. In particular, it is necessary to understand how the acceleration of the atmosphere affects the local state variables such as pressure $p$, density $\rho$ and temperature $T$.

Consider a plane-parallel atmosphere divided horizontally into two regions. The upper moves downwards with velocity $\dot{\boldsymbol{r}}_{1}$, the lower moves upwards (velocity $\dot{\boldsymbol{r}}_{2}$ ). The downward flow is thus accelerated at a compression front where it meets the upward flow. The position of the front moves upwards (velocity $\boldsymbol{u}$ ) as material flows through it (Fig. 14). To a first approximation, $\dot{\boldsymbol{r}}_{1} \approx \dot{\boldsymbol{r}}(\phi=0.1)$ and $\dot{\boldsymbol{r}}_{2} \approx \dot{\boldsymbol{r}}(\phi=0.2)$. The compression wave velocity can be obtained from the time $\delta t$ taken for the line-forming region to accelerate from $\dot{\boldsymbol{r}}_{1}$ to $\dot{\boldsymbol{r}}_{2}$ and the pressure scale height $H$. Taking the line formation region to be at optical depth $\tau \approx 0.1$ the local temperature $T \approx 20000 \mathrm{~K}$. With $\log g \approx 3.5$ and the mean atomic mass $\mu \approx 2$ for singly-ionized helium,

$H=\frac{p}{g \rho}=\frac{k T}{g \mu m_{\mathrm{H}}} \approx 2.6 \times 10^{3} \mathrm{~km}$

$\left(p, \rho, k\right.$ and $m_{\mathrm{H}}$ represent pressure, density, Boltzmann's constant and the mass of the hydrogen atom, respectively). Considering that $90 \%$ of the acceleration lasts just 0.05 cycle (Fig. 10), $\delta t \approx 500$ s. Hence

$u \sim H / \delta t \sim 5 \mathrm{~km} \mathrm{~s}^{-1}$.

Assuming the ratio of specific heats $\gamma=5 / 3$ for convenience, the local sound speed is given by

$c_{1}=\left(\frac{\gamma p}{\rho}\right)^{1 / 2}=\left(\frac{\gamma k T}{\mu m_{\mathrm{H}}}\right)^{1 / 2} \approx 12 \mathrm{kms}^{-1}$.

Paper III obtained similar values and argued essentially that no shock would occur because the speed of the compression front was less than the sound speed. The authors failed to appreciate that the crucial parameter is the gas velocity relative to the compression front. Transforming to the frame of the compression front (see Fig. 14),

$\boldsymbol{v}_{1}=\boldsymbol{u}+\left(\dot{\boldsymbol{r}}_{1}-\dot{\boldsymbol{r}}_{2}\right), \boldsymbol{v}_{2}=\boldsymbol{u}$.

The Mach number of the gas flowing into the front is then

$M_{1}=\frac{v_{1}}{c_{1}}=\frac{104}{12} \approx 8$.

Thus the fluid flow into the front is supersonic and the development of a shock is inevitable. Properties of the atmosphere behind the shock can be deduced from the Rankine-Hugoniot conditions (cf. Shore 1992, p.106f.) and give the Mach number, gas temperature, pressure, and density in terms of gas properties in front of the shock. Thus, with $\gamma=5 / 3$,

$$
\begin{aligned}
M_{2}^{2} & =\frac{(\gamma-1) M_{1}^{2}+2}{\left.2 \gamma M_{1}^{2}-(\gamma-1)\right)} \\
\Rightarrow M_{2} & =\left(\frac{M_{1}^{2}+3}{5 M_{1}^{2}-1}\right)^{1 / 2} \approx 0.46 \\
\frac{T_{2}}{T_{1}} & =\frac{\left(2 \gamma M_{1}^{2}-(\gamma-1)\right)\left((\gamma-1) M_{1}^{2}+2\right)}{\left((\gamma+1) M_{1}\right)^{2}} \\
& =\frac{\left(5 M_{1}^{2}-1\right)\left(M_{1}^{2}+3\right)}{16 M_{1}^{2}} \approx 21 \\
\frac{p_{2}}{p_{1}} & =\frac{2 \gamma M_{1}^{2}-(\gamma-1)}{\gamma+1}=\frac{1}{4}\left(5 M_{1}^{2}-1\right) \approx 80 \\
\frac{\rho_{2}}{\rho_{1}} & =\frac{(\gamma+1) M_{1}^{2}}{(\gamma-1) M_{1}^{2}+2}=\frac{4 M_{1}^{2}}{M_{1}^{2}+3}=\frac{v_{1}}{v_{2}}=3.8 .
\end{aligned}
$$

This last result lies close to the strong shock limit $\rho_{2} / \rho_{1} \sim 4$. The shock locally heats the fluid to $\sim 400000 \mathrm{~K}$. Adiabatic expansion will reduce this relatively quickly, but 
it is not expected that hydrostatic and thermal equilibrium will be achieved immediately after passage of the shock front.

From this thumbnail calculation it may be seen that the dynamical consequences of the pulsations in V652 Her will be profound for at least part of the pulsation cycle, and maybe throughout. In deeper layers, where the sound speed is higher, the compression and heating factors will be weaker, but the radiative cooling times will also be longer. Model atmospheres which consider radiation hydrodynamics will need to be constructed.

An interesting consequence of this calculation is that temperatures $\sim 4 \times 10^{5} \mathrm{~K}$ in the shock front may produce an X-ray flash once every pulsation cycle. Further calculations will demonstrate whether this could be detected with current X-ray telescopes. Recombination lines might also be expected to appear for a short interval each cycle; none have been observed to date.

A more disturbing consequence is that, while it is evident that current methods of spectroscopic analysis break down around minimum radius, the validity of the hydrostatic approximation used in the spectroscopic analysis during the remainder of the pulsation cycle must come under scrutiny. The dynamical timescale may be estimated from free-fall through a pressure scale height,

$t_{\mathrm{dyn}}=\sqrt{\frac{2 H}{g}} \approx 300 \mathrm{~s}$,

while the thermal relaxation time may be estimated from the sound-speed

$t_{\mathrm{th}}=\frac{H}{c_{1}} \approx 200 \mathrm{~s}$.

Both are short compared with the pulsation period ( $\approx 9000 \mathrm{~s})$ so that, according to these estimates, approximate hydrostatic equilibrium should be re-established shortly after the shock front has passed through the atmosphere.

Further diagnostics of the shock passage are expected to be visible in the infrared, where He I and Paschen lines are formed at higher levels in the atmosphere. Highresolution observations with $8 \mathrm{~m}$ class telescopes are now capable of resolving the line profiles with sufficient temporal resolution to allow such a study to be made.

\subsection{Non-equilibrium phenomena in V652 Her and other pulsating stars}

The occurrence of non-equilibrium phenomena should, in fact, be expected from both observational and theoretical consideration of pulsations in other stellar classes. The doubling of metallic lines was first seen in W Vir (Sanford 1952) and explained by the passage of an upward propagating shock front (Schwarzschild 1952) splitting the line forming region into two components moving in opposite directions. In terms of stellar dimensions and pulsational characteristics, the variables most similar to V652 Her are possibly the RR Lyraes and the $\beta$ Cepheids. H-absorption line doubling during rising light in RR Lyr is well known (Sanford 1949; Preston et al. 1965); metallic line doubling in RR Lyr has also been established (Preston et al. 1965; Chadid \& Gillet 1996a). Very detailed observations of these lines have enabled a variety of phenomena within the pulsating atmosphere, including turbulence and shocks, to be studied (e.g. Chadid \& Gillet 1996b; Fokin et al. 1999).

A feature of the observations of line doubling in RR Lyr was that a resolving power of 42000 , signal-tonoise ratio $>50$ and a time resolution close to $1 \%$ of the $13.6 \mathrm{~h}$ pulsation period were required (Chadid \& Gillet 1996a). Moreover the intrinsic line widths, due to heavier ions and lower $T_{\text {eff }}$, are much smaller than in V652 Her, making line doubling naturally easier to detect. Therefore the evidence for line broadening, at least, in our much lower resolution observations of V652 Her points to the existence of non-equilibrium phenomena worthy of further study.

Other non-equilibrium phenomena include differences in amplitude or phase lags beween the radial velocity curves measured from metal lines and neutral or ionized helium lines. Such phase lags are well known in $\beta$ Cepheids (the Van Hoof effect, van Hoof \& Struve 1953) and in RR Lyr (Mathias et al. 1995). These have been looked for in the current dataset but so far without success.

There is marginal evidence for small cycle-to-cycle changes in the radial velocity (Paper III) and light curve (Kilkenny et al. 1996) of V652 Her. The long-term coverage of either has so far been insufficient to detect anything like the Blazhko effect (Blazhko 1907), but a corollary may be drawn with irregularities in the single-line radial velocity curve for RR Lyr (Chadid 2000). Two explanations were proposed for the latter, one being a connection with the Blazhko effect and the second being a dynamical interaction between the highest and lowest layers of the atmosphere mediated by strong outward shock waves.

Clearly, it is too early to draw direct comparisons between the pulsations in V652 Her and other stellar classes. However the prospects for exploring the physics of stellar pulsations in a different area of parameter space are good.

\section{The Mass of V652 Her}

\subsection{Comparison with previous analyses}

The new measurements of $r_{0}$ and $M$ for V652 Her are clearly smaller than those given before (Paper II). Reasons include an improved projection factor which has increased all of the derived radii by $\sim 10 \%$. All mass estimates are reduced because the effective surface gravity has been reduced by $\sim 0.35$ dex relative to Paper II. The new phasedependent measurements of $\log g$ are far more reliable than previous results except at phases close to minimum radius and automatically give a substantial reduction in mass, irrespective of radius. 


\subsection{Which is the correct mass?}

Table 4 lists four alternative sets of mass, radius and effective temperature for V652 Her. A major conclusion of the present work is that the current model atmospheres are not able to provide consistent $T_{\text {eff }}$ from optical spectroscopy and total flux methods, so that it is probably premature to draw a final conclusion. It would be inappropriate to use the radii and masses measured from optical spectroscopy and $V$-band photometry alone because the corresponding models do not have sufficient flux compared with the ultraviolet observations. Moreover, these masses $\left(0.18 \pm 0.05 M_{\odot}\right)$ appear to be unphysically low.

The mass measured by the total flux method $(0.59 \pm$ $\left.0.18 M_{\odot}\right)$ and that reported in Paper II $\left(0.7_{-0.3}^{+0.4} M_{\odot}\right)$ are within the error estimates of each other, which is reassuring. Paper IV refined the latter to $0.69_{-0.12}^{+0.15} M_{\odot}$ by reducing the measurement error on $\log g$, but this has little bearing on the current question.

For the present, it seems reasonable to regard the measurements of Paper II and those presented here from the total flux method as likely extremes. A mass of $0.59 M_{\odot}$ with a random error of $\pm 0.18 M_{\odot}$ and a systematic error of $\pm 0.1 M_{\odot}$ would represent the current observational data and model atmospheres.

The mass for V652 Her $\left(0.59 M_{\odot}\right)$ should be compared with that deduced for the pulsating helium star BX Cir (=LSS 3184, Woolf \& Jeffery 2000) with $M=$ $0.42 \pm 0.12 M_{\odot}$. The pulsation periods for BX Cir and V652 Her are almost identical. The average radii of both stars were measured using almost identical methods, giving $2.31 \pm 0.01 R_{\odot}$ for $\mathrm{V} 652$ Her and $2.31 \pm 0.10 R_{\odot}$ for BX Cir. The original mass measurement for BX Cir had seemed small compared with that expected from previous work on V652 Her. Now the close correspondence of their radii and their pulsation periods encourages us to suppose that the mass measurements are still dominated by systematic effects. By virtue of the period mean-density relation for radially pulsating stars, the masses of BX Cir and V652 Her must be very similar.

Regarding the evolutionary origin of V652 Her, this value or range of possible values for the mass, together with the corresponding radii and effective temperaures and the measured chemical composition are quite appropriate for the merged binary white dwarf model (Saio \& Jeffery 2000). While a hydrodynamical model with $0.7 M_{\odot}$ (Fadeyev \& Lynas-Gray 1996) matches the pulsation properties very well, we are currently studying (Montañés Rodriguez \& Jeffery 2001b) a more extended family of such models with different masses and radii. These have very similar pulsation properties including period, and light and velocity curve amplitude and shape.

One cause for concern is that the total flux method depends on relatively few IUE measurements which are both noisy and poorly distributed in phase. Omitting just one of these can have a major effect on the final mass. More frequent and less noisy ultraviolet measurements are crucial to a more accurate measurement. Similarly, improvements in the model atmospheres are prerequisite to significant further progress.

Acknowledgements. This research was supported through grants in aid from the Departments of Education and of Culture, Arts and Leisure in Northern Ireland, and through a UK Particle Physics and Astronomy Research Council (PPARC) grant /PPA/G/S/1998/00019 to the Armagh Observatory. Dr Dave Kilkenny (SAAO) kindly provided the data from the visual light curve for V652 Her. Dr Tom Kerr (UKIRT) kindly reduced the infrared spectrum. Discussions with Prof. Philip Dufton concerning the implementation of He I line broadening data were much appreciated. As referee, Prof. Ulrich Heber made many valuable suggestions which have been incorporated. Much of the analysis software was developed from programs provided orginally through CCP7, a PPARC project for the "Analysis of Astronomical Spectra".

\section{Appendix A: Computer programs for analysing stellar spectra}

The availability of spectra covering a large wavelength range with good spectral and temporal resolution makes an attempt to measure the instantaneous effective temperature $\left(T_{\text {eff }}\right)$ and surface gravity $(\log g)$ throughout the pulsation cycle attractive. In contrast, the previous spectral analysis (Paper IV) only considered an "average spectrum" integrated over the pulsation cycle, with a resolution and $S / N$ poorer than that of each individual spectrum considered here.

Under ideal circumstances, such an analysis should take the dynamical nature of the atmosphere into account. The primary argument in favour of using the hydrostatic approximation is that it should be shown to be inadequate before investing the effort required to construct dynamical model atmospheres. The assumptions of hydrostatic and thermal equilibrium will be re-examined later (Sect. 7).

With these considerations, the following tools were adopted or developed to analyse the high-quality spectra of V652 Her.

\section{A.1. Sterne}

We have used model atmospheres calculated using the code STERne (Jeffery \& Heber 1992). The code assumes hydrostatic, radiative and local thermodynamic equilibrium and plane-parallel geometry. Continuous opacities are nearly the same as those adopted by Kurucz (1979), with the addition of carbon and nitrogen from Peach (1970), while line opacities are accounted for using an opacity distribution function computed for a hydrogendeficient mixture by (Möller 1990) from the Kurucz \& Petryemann (1975) list of 265000 lines, in the same manner as adopted in Kurucz's stellar atmosphere program ATLAS6 (cf. Kurucz 1979). Radiative transfer is solved using the Feautrier scheme (Feautrier 1964) and temperature correction is achieved using the Unsöld-Lucy procedure (Lucy 1964). The plane-parallel approximation is justified since the pressure scale height in the atmosphere 


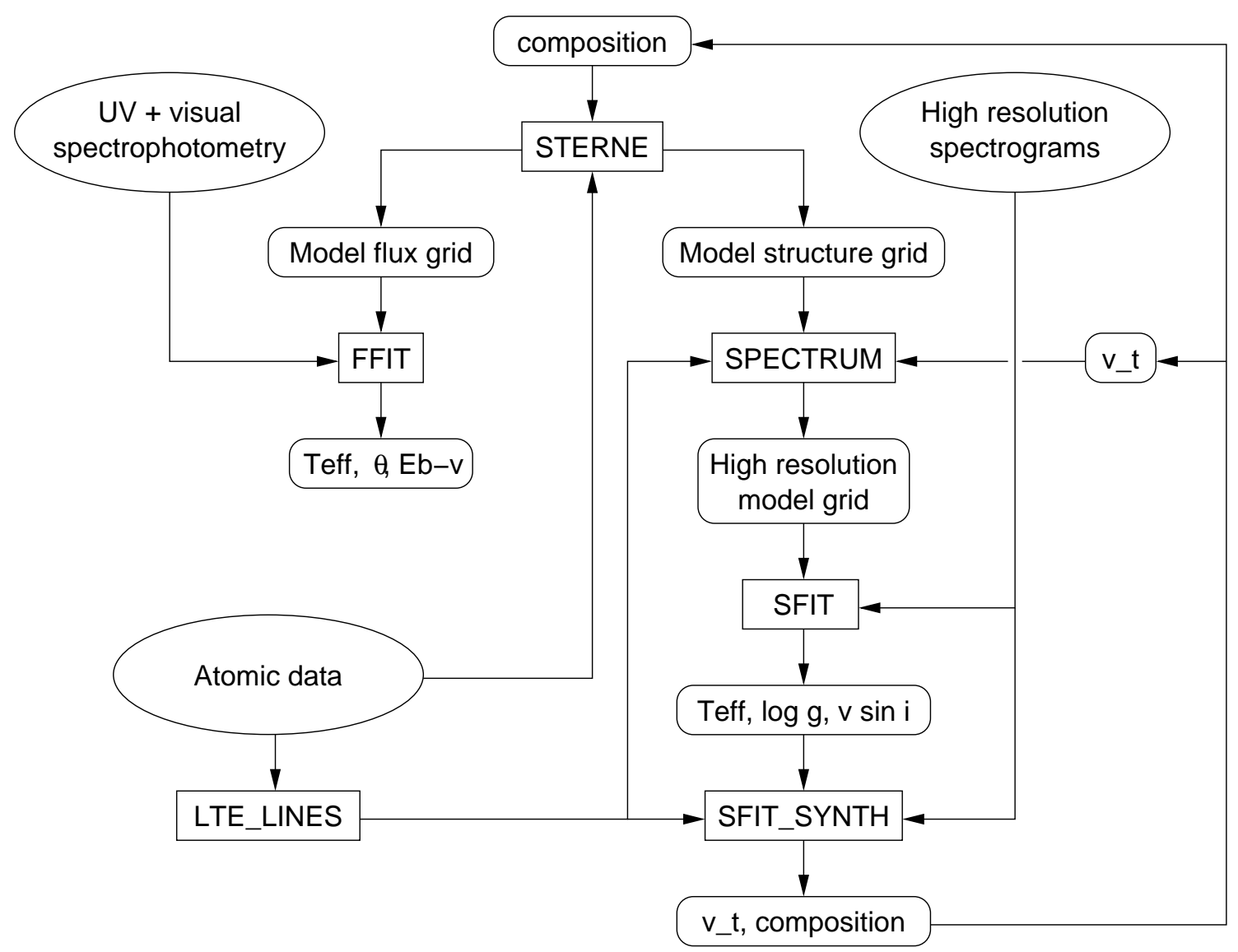

Fig. A.1. Block diagram illustrating the procedures (boxes), inputs (ellipses) and outputs (oval boxes) used in the analysis of high-resolution optical spectra and broad-band spectrophotometry of V652 Her.

of V652 Her is small $(<0.2 \%)$ compared to the stellar radius, even for the lowest gravity models considered.

The emergent flux distributions, sampled at 342 wavelengths between $229 \AA$ and $20 \mu \mathrm{m}$, were used to measure effective temperatures and angular diameter from ultraviolet and visual photometry. The model structures describe temperature, pressure and electron density as a function of optical depth on a grid of fifty depth points, and were used as input for the spectral synthesis calculations described below (Sect. 4.3).

\section{A.2. Ffit}

The method adopted here to measure effective temperature, angular diameter and insterstellar extinction was to fit the reddened theoretical flux distribution

$\phi_{\lambda}\left(E_{B-V}, T_{\text {eff }}, \theta\right)=\theta^{2} f_{\lambda}\left(T_{\text {eff }}\right) A_{\lambda}\left(E_{B-V}\right)$

to the observed flux distribution $F_{\lambda}$ using the method of $\chi^{2}$ minimization. The flux distributions are first resampled to the resolution of the theoretical flux distributions, this being lower. The specific extinction $A_{\lambda}$ is taken from the average Galactic extinction law due to Seaton (1979). In computing

$\chi^{2}=\Sigma_{\lambda} \frac{\left(F_{\lambda}-\phi_{\lambda}\right)^{2}}{\sigma_{\lambda}^{2}}$, $\sigma_{\lambda}^{2}$ are the variances of the binned fluxes. The errors associated with the best fit parameters $x_{i}$ are given by the diagonal elements $\left(\alpha^{-1}\right)_{i i}$ of the inverse of the covariance matrix $\alpha$, whose elements are given by

$\alpha_{i j}=\Sigma_{\lambda}\left(\frac{\partial \phi_{\lambda}}{\partial x_{i}} \frac{\partial \phi_{\lambda}}{\partial x_{j}} / \sigma_{\lambda}^{2}\right)$

The minimum in the multi-dimensional $\chi^{2}$ surface was located using the downhill simplex method (Nelder \& Mead 1965), implemented using a variant of the algorithm AmoebA (Press et al. 1989). The method was proven to give identical results to an independently developed brute-force algorithm (cf. Jeffery et al. 2000). The principal difference between our version of AMOEBA and that published by Press et al. is that ours passes both the free parameters and the observed spectrum to the function to be minimized. In this case, the function is $\chi^{2}$ (cf. CHISQ, Press et al. 1989).

In applying FFIT, care was taken to ensure that the normalization of theoretical and observed fluxes was carried out at optical wavelengths, otherwise small errors in $E_{B-V}$ and $T_{\text {eff }}$ would have led to major errors in $\theta$. Although $E_{B-V}$ may be found as an independent parameter in each fit, the final results were constrained to have a single "average" value of $E_{B-V}$ for repeated observations of V652 Her. 
FFIT was tested by application to a spectrum of Vega used for calibration of the Hubble Space Telescope (Colina et al. 1996). The calibration spectrum was restricted to match the IUE wavelength interval, and supplemented with $U B V R I$ photometry, assuming $U=$ $B=V=R=I=0.0$. The photometry was converted to flux units with the calibration constants $C_{\lambda}=-20.94,-20.51,-21.12,-21.89,-22.70$, respectively. Using a grid of ATLAS9 model atmospheres (Kurucz 1991) with $\log g=4.0$ and $[\mathrm{Fe} / \mathrm{H}]=-0.5$, FFIT yielded $T_{\text {eff }}=9617 \pm 24 \mathrm{~K}, \theta=7.67 \pm 0.02 \times 10^{-9}$ radians and $E_{B-V}=0.000 \pm 0.004$ (formal errors). Both $T_{\text {eff }}$ and $\theta$ differ by less than $1 \%$ from the result reported by Castelli \& Kurucz (1994).

\section{A.3. Spectrum}

The formal solution code SPECTRUM was written originally by Prof P. L. Dufton and extended by Drs. D. J. Lennon and E. S. Conlon at Queen's University Belfast. It was adapted for use with $\mathrm{H}$-deficient mixtures and considerably extended by one of us (CSJ). From a given model atmosphere and a line list including $g f$-values, radiative and collisional broadening constants and excitation energies, SPECTRUM can now compute synthetic spectra over large wavelength intervals and for many absorption lines. The usual output product is the normalized spectrum

$s_{\lambda}\left(T_{\mathrm{eff}}, \log g, v_{\mathrm{t}}, n_{i}, i=1, \ldots\right)=\frac{f_{\lambda}}{f_{\mathrm{c}}}$

computed as a function of $T_{\text {eff }}, \log g$, microturbulent velocity $v_{\mathrm{t}}$, and chemical composition given as the relative abundance by number $n_{i}$ of species $i$. In addition, the total emergent flux $f_{\lambda}$, the continuum flux $f_{\lambda c}$ and specific intensities $I_{\lambda \mu}$ may also be obtained explicitly.

Metal line profiles are computed as Voigt functions, including thermal and Doppler broadening due to microturbulence, and radiative and collisional broadening. Hydrogen lines are computed using broadening tables from Lemke (1997) and based on broadening theory by Vidal et al. (1973). Neutral helium line profiles for $\operatorname{He}$ I $\lambda 4471,4026,4922$ and $4388 \AA$ are computed from broadening tables by Barnard et al. (1969, 1974, 1975). Profiles for He I $\lambda 4144$ and $4009 \AA$ are computed from broadening theory by Gieske \& Griem (1969) and by Dimitrijevic \& Sahal-Brechot (1984), whilst the remainder are computed as Voigt profiles. Line profiles for ionized helium are computed from broadening tables by Schöning \& Butler (1989).

The model atmopheres grid described above was used to generate grids of synthetic spectra comprising some 1100 absorption lines on the interval 4250-4950 A.

\section{A.4. Lte_lines}

Atomic data for metal lines comes from a large variety of sources; much of the data has been tested extensively in analyses of B stars. A description of the atomic database LTE_LINES, which includes a utility for selecting appropriate ions to include in the linelist, has been provided by Jeffery (1991) and is also available on the WWW at http://www . arm.ac.uk/ csj/linelists.html. Data for some lines have been obtained from linelists provided on CD-ROM (Kurucz 1993) and from the Vienna Atomic Line Database (Piskunov et al. 1995). Where both experimental or theoretical broadening constants are unavailable, classical values are used.

\section{A.5. Sfit}

Our goal was to estimate the various parameters of the stellar atmosphere (effective temperature, surface gravity, chemical composition, etc.) by finding the combination that produces a theoretical spectrum which most closely resembles the observed spectrum.

In addition to the natural broadening of spectral lines in the stellar atmosphere by processes described above, additional broadening processes must be considered. These processes are applied to the synthetic spectra before comparison with the observed spectrum and include:

- Instrumental broadening $I(\Delta \lambda)$ : each spectrum is convolved with a Gaussian having a $F W H M \Delta \lambda$ equal to the instrumental resolution measured from the comparison lamp emission lines and being $0.46 \AA$.

- Rotation broadening $V(v \sin i, \beta)$ : each spectrum is convolved with the rotation broadening function (Unsöld 1955; Dufton 1972), assuming a limb darkening factor $\beta=1.5$. The projected rotation velocity $v \sin i$ is a free parameter of the model.

- Acceleration broadening $A(\delta v)$ : the change in radial velocity $\delta v$ during each $100 \mathrm{~s}$ exposure of V652 Her is typically $1 \mathrm{~km} \mathrm{~s}^{-1}$ and around minimum radius rises to $\sim 10 \mathrm{~km} \mathrm{~s}^{-1}$. An experiment was performed in which each spectrum was convolved with a boxcar of full width equal to the acceleration. In practise, more consistent solutions were found without this broadening being applied.

- Projection broadening $P(v-\bar{v})$ : the projection of the spherical expansion onto the line of sight convolved with the specific intensity of the emergent flux distorts and broadens the line profile as a function of phase, as discussed elsewhere (Montañés Rodriguez \& Jeffery 2001a). The asymmetries predicted for V652 Her, when convolved with other effects were found to be negligible. We have been unable to detect any asymmetry in the line profile as a continuous function of pulsation phase.

For a given observation, an optimum fit in $T_{\text {eff }}, \log g$ and $v \sin i$ was obtained by minimizing $\chi^{2}$, the weighted square residual between the normalized observed spectrum $S_{\lambda}=F_{\lambda} / F_{\mathrm{c}}$ and the theoretical spectrum

$$
\begin{aligned}
s_{\lambda}^{\prime}= & s_{\lambda}\left(T_{\mathrm{eff}}, \log g\right) \otimes I(\Delta \lambda) \otimes V(v \sin i, \beta) \\
& \otimes A(\delta v) \otimes P(v-\bar{v}) .
\end{aligned}
$$


The model spectrum for arbitrary $T_{\text {eff }}, \log g$ was obtained using a two dimensional polynomial interpolation in the discrete model grid. For accuracy, the algorithm POLIN2 (Press et al. 1989) was adopted. The $\chi^{2}$-minimization was carried out using the new-variant algorithm AMOEBA described above. The method was proven to be robust by repeated applications using different model grid spacings and starting values.

In the construction of $\chi^{2}$, each wavelength point was given a weight $w_{\lambda}=1 / \sigma_{\lambda}$, defined as the standard deviation about the mean flux in line-free regions, where $\sigma_{\lambda} \sim 0.01$. Since the cores of strong He I lines still consistently fail to provide agreement between theory and observation, they were partially excluded from the fit by assigning a lower weight to the corresponding wavelength points $\left(\sigma_{\lambda}=0.1\right)$.

In any such fitting procedure, the normalization of the observed spectrum can be of crucial importance (cf. Paper IV). The initial normalization was carried out by fitting a high-order polynomial to apparent sections of "continuum". The presence of even a few weak lines can mean that this procedure sets the "continuum" too low. We adopted a single improvement iteration (cf. Jeffery et al. 1998). Having found a best-fit $s_{\lambda}^{\prime}$, the ratio $S_{\lambda} / s_{\lambda}^{\prime}$ was trimmed to exclude values more than $5 \%$ from continuum and convolved with a low-pass Gaussian filter of $F W H M 18 \AA$. This provided a renormalization function for each spectrum which departed not more than $1 \%$ from unity, with a standard deviation of $\lesssim 0.5 \%$. The renormalization had no effect on individual line profiles.

An extension of the above code allows for the variation of one other parameter, the helium abundance $n_{\mathrm{He}}$ for example, was not used in the current investigation but is noted for completeness. The grid of model atmospheres and synthetic spectra may be extended to three dimensions $T_{\text {eff }}, \log g$ and the fractional helium abundance $n_{\mathrm{He}}$. Interpolation is carried out first in $n_{\mathrm{He}}$ to obtain a subgrid in which the two-dimensional interpolation for $T_{\text {eff }}$ and $\log g$ can be carried out as above.

\section{A.6. Sfit_synth}

If $T_{\text {eff }}, \log g$ and $v \sin i$ are known, the composition of a star may be obtained by adjusting the abundances of the different atomic species which contribute to the absorption spectrum, together with the microturbulent velocity $\left(v_{\mathrm{t}}\right)$, so that the theoretical spectrum matches the observed spectrum. This can be achieved by minimizing the same weighted $\chi^{2}$ residual between observed and theoretical spectrum as used by SFIT. However, in the present case, the number of free parameters, namely the abundances of $\mathrm{H}, \mathrm{C}, \mathrm{N}, \mathrm{O}, \mathrm{Al}, \mathrm{Si}, \mathrm{P}, \mathrm{S}, \mathrm{Ne}, \mathrm{Mg}$, and Fe and $v_{\mathrm{t}}$, was so large that precomputing multidimensional grids of theoretical spectra would have been prohibitive. The solution adopted was to compute synthetic spectra in real time as demanded by the $\chi^{2}$ minimization procedure, AMOEBA.
The same line-broadening (rotation, instrumental, acceleration, projection) could be introduced as before.

In principle, this code SFIT_SYNTH could solve simultaneously for as many parameters as are required. It was found to be more practical to restrict each run of the code to between two and four parameters. A single model atmophere is assumed as input, so that $T_{\text {eff }}, \log g$ and $v \sin i$ remain fixed for a given solution. Then the code would attempt to solve for $v_{\mathrm{t}}$ and one chemical abundance together, or for two or three chemical abundances simultaneously, whilst other chemical abundances were kept fixed.

\section{A.7. Operation of the Programs}

Given the physical assumptions outlined above and either a high-resolution optical spectrum or low-resolution spectrophotometry covering ultraviolet and visual wavelengths (at least), these programs allow us to drive various physical quantities in an objective manner. The outputs are either $T_{\text {eff }}, \log g, v \sin i, v_{\mathrm{t}}$ and chemical composition from a high-resolution spectrum, or $T_{\text {eff }}, \theta$ and $E_{B-V}$ from spectrophotometry. A block diagram illustrating the procedures, inputs and outputs, is shown in Fig. A.1. Note that it is essential to ensure that the composition used as input to the model atmospheres is consistent with that derived as output from the spectral analysis. The latter critically affects the background opacities and hence the temperature stratification in the former, especially where hydrogen is absent from the stellar atmosphere. Therefore a few iterations may be necessary before a final solution is achieved.

\section{References}

Barnard, A. J., Cooper, J., \& Shamey, L. J. 1969, A\&A, 1, 28 Barnard, A. J., Cooper, J., \& Smith, E. W. 1974, JQSRT, 14, 1025

Barnard, A. J., Cooper, J., \& Smith, E. W. 1975, JQSRT, 15, 429

Blazhko, S. 1907. Astr. Nachr., 175, 325

Bohlin, R. C., Savage, B. D., \& Drake, J. F. 1978, ApJ, 224, 132

Castelli, F., \& Kurucz, R. L. 1994, A\&A, 281, 817

Chadid, M. 2000, A\&A, 359, 991

Chadid, M., \& Gillet, D. 1996a, A\&A, 308, 481

Chadid, M., \& Gillet, D. 1996b, A\&A, 315, 475

Colina, L., Bohlin, R., \& Castelli, F. 1996, HST instrument science report, CAL/SCS-008

Dimitrijevic, M. S., \& Sahal-Brechot, S. 1984, JQSRT, 31, 301

Dimitrijevic, M. S., \& Sahal-Brechot, S. 1989, Bull. Obs. Astr. Belgrade, 141, 57

Dimitrijevic, M. S., \& Sahal-Brechot, S. 1990, A\&AS, 82, 519

Drilling, J. S., Jeffery, C. S., \& Heber, U. 1998, A\&A, 329, 1019

Dufton, P. L. 1972, A\&A, 16, 301

Fadeyev, Yu. A., \& Lynas-Gray, A. E. 1996, MNRAS, 280, 427

Feautrier, P. 1964, C. R. Acad. Sci. Paris, 258, 3189

Fokin, A. B., Gillet, D., \& Chadid, M. 1999, A\&A, 344, 930

Gieske, H. A., Griem, H. R. 1969, ApJ, 157, 963

Grevesse, N., Noels, A., \& Sauval, A. J. 1996, Cosmic abundances, ed. S. S. Holt, \& G. Sonneborn, ASP Conf. Ser., 99,117 
Groenewegen, M. A. T., \& Lamers, H. J. G. L. M. 1989, A\&AS, 79, 359

Harrison, P. M., \& Jeffery, C. S. 1997, A\&A, 323, 177

Heber, U. 1983, A\&A, 118, 39

Hill, P. W., Kilkenny, D., Schönberner, D., \& Walker, H. J. 1981, MNRAS, 197, 81 (Paper I)

Hubeny, I., Lanz, T., \& Jeffery, C. S. 1994, CCP7 Newslett., 20,30

Iben, I., Jr., Kaler, J. B., Truran, J. W., \& Renzini, A. 1983, ApJ, 264, 605

Iben, I., Jr., \& MacDonald, J. 1995, White Dwarfs, Lecture Notes in Physics, ed. D. Koester, \& K. Werner (SpringerVerlag, Berlin Heidelberg New York), 443, 48

Iben, I., Jr., \& Tutukov, A. 1985, ApJS, 58, 661

Jeffery, C. S. 1991, Newsletter on Analysis of Astronomical Spectra, 16, 17

Jeffery, C. S., Hamill, P. J., Harrison, P. M., \& Jeffers, S. V. 1998, A\&A, 340, 476

Jeffery, C. S., \& Harrison, P. M. 1997, A\&A, 323, 393

Jeffery, C. S., \& Heber, U. 1992, A\&A, 260, 133

Jeffery, C. S., \& Hill, P. W. 1986, MNRAS, 221, 975 (Paper III)

Jeffery, C. S., Hill, P. W., \& Heber, U. 1999, A\&A, 346, 491 (Paper IV)

Jeffery, C. S., Starling, R. L. C., Hill, P. W., \& Pollacco, D. 2000, MNRAS, in press

Kilkenny, D. 1988, MNRAS, 232, 377

Kilkenny, D., \& Lynas-Gray, A. E. 1982, MNRAS, 198, 873

Kilkenny, D., \& Lynas-Gray, A. E. 1984, MNRAS, 208, 673

Kilkenny, D., Lynas-Gray, A. E., \& Roberts, G. 1996, MNRAS, 283, 1349

Kurucz, R. L. 1979, ApJS, 40, 1

Kurucz, R. L. 1988, in IAU Trans., ed. M. McNally (Kluwer, Dordrecht), 20B , 168

Kurucz, R. L., \& Petryemann, E. 1975, A Table of Semiempirical gf Values, SAO Special Report, 362 (Cambridge, Massachussets 02138)

Kurucz, R. L. 1993, SAO KURUCZ CD-ROM, No. 18

Kurucz, R. L. 1991, in Stellar Atmospheres: Beyond Classical Models, 441, ed. L. Crivellari, I. Hubeny, \& D. G. Hummer, NATO ASI Series Vol. C341 (Kluwer, Dordrecht)

Landolt, A. U. 1975, ApJ, 196, 787
Lynas-Gray, A. E., Schönberner, D., Hill, P. W., \& Heber, U. 1984, MNRAS, 209, 387 (Paper II)

Lemke, M. 1997, A\&AS, 122, 285

Lucy, L. 1964, SAO Special Report, 167, 93

Mathias, P., Gillet, D., Fokin, A. B., \& Chadid, M. 1995, A\&A, 298, 843

Moore, C. E. 1945, Contr. Princeton Univ. Obs., 20 (Princeton, New Jersey)

Möller, R. U. 1990, D. Th., Universität Kiel

Montañès Rodriguez, P., \& Jeffery, C. S. 2001a, A\&A, in press

Montañès Rodriguez, P., \& Jeffery, C. S. 2001b, A\&A, in preparation

Nelder, J. A., \& Mead, R. 1965, Computer Journal, 7, 308

Parsons, S. B. 1972, ApJ, 174, 57

Piskunov, N. E., Kupka, F., Ryabchikova, T. A., Weiss, W. W., \& Jeffery, C. S. 1995, A\&AS, 112, 525

Press, W. H., Flannery, B. P., Teukolsky, S. A., \& Vetterling, W. T. 1989, Numerical Recipes: The Art of Scientific Computing (Cambridge University Press)

Preston, G. W., Smak, J., \& Paczynski, B. 1965, ApJS, 12, 99

Rodriguez-Pascual, P. M., Gonzalez-Riestra, R., Schartel, N., \& Wamsteker, W. 1999, A\&AS, 139, 183

Saio, H. 1993, MNRAS, 260, 465

Saio, H., \& Jeffery, C. S. 2000, MNRAS, 313, 671

Sanford, R. F. 1949, ApJ, 109, 208

Sanford, R. F. 1952, ApJ, 116, 331

Schöning, T., \& Butler, K. 1989, A\&AS, 78, 51

Schrijver, H. 1997, The Hipparcos and Tycho Catalogue, 8, European Space Agency

Schwarzschild, M. 1952, Transaction of the IAU, VIII, 8111

Seaton, M. J. 1979, MNRAS, 187, 73P

Shapley, H., \& Nicholson, S. B. 1919, Proc. Nat. Acad. Sci., 5, 417.

Shore, S. N. 1992, An Introduction to Astrophysical Hydrodynamics (Academic Press)

Unsöld, A. 1955, Physik der Sternatmosphären, 2nd ed. (Berlin: Springer-Verlag)

van Hoof, A., \& Struve, O. 1953, PASP, 65, 158

Vidal, C. R., Cooper, J., \& Smith, E. W. 1973, ApJS, 25, 37

Webbink, R. F. 1984, ApJ, 277, 355

Woolf, V. M., \& Jeffery, C. S., A\&A, 358, 1001 